\title{
Integrable sigma models and 2-loop RG flow
}

\author{
Ben Hoare, ${ }^{a}$ Nat Levine ${ }^{b}$ and Arkady A. Tseytlin ${ }^{b, 1}$ \\ ${ }^{a}$ Institut für Theoretische Physik, ETH Zürich, \\ Wolfgang-Pauli-Strasse 27, 8093 Zürich, Switzerland \\ ${ }^{b}$ Blackett Laboratory, Imperial College, \\ London SW7 2AZ, U.K. \\ E-mail: bhoare@ethz.ch, n.levine17@imperial.ac.uk, \\ tseytlin@imperial.ac.uk
}

ABSTRACT: Following arXiv:1907.04737, we continue our investigation of the relation between the renormalizability (with finitely many couplings) and integrability in $2 \mathrm{~d} \sigma$ models. We focus on the " $\lambda$-model," an integrable model associated to a group or symmetric space and containing as special limits a (gauged) WZW model and an "interpolating model" for non-abelian duality. The parameters are the WZ level $k$ and the coupling $\lambda$, and the fields are $g$, valued in a group $G$, and a $2 \mathrm{~d}$ vector $A_{ \pm}$in the corresponding algebra. We formulate the $\lambda$-model as a $\sigma$-model on an extended $G \times G \times G$ configuration space $(g, h, \bar{h})$, defining $h$ and $\bar{h}$ by $A_{+}=h \partial_{+} h^{-1}, A_{-}=\bar{h} \partial_{-} \bar{h}^{-1}$. Our central observation is that the model on this extended configuration space is renormalizable without any deformation, with only $\lambda$ running. This is in contrast to the standard $\sigma$-model found by integrating out $A_{ \pm}$, whose 2-loop renormalizability is only obtained after the addition of specific finite local counterterms, resulting in a quantum deformation of the target space geometry. We compute the 2-loop $\beta$-function of the $\lambda$-model for general group and symmetric spaces, and illustrate our results on the examples of $\mathrm{SU}(2) / \mathrm{U}(1)$ and $\mathrm{SU}(2)$. Similar conclusions apply in the non-abelian dual limit implying that non-abelian duality commutes with the RG flow. We also find the 2-loop $\beta$-function of a "squashed" principal chiral model.

KEywords: Integrable Field Theories, Renormalization Group, Sigma Models

ARXIV EPRINT: 1910.00397

\footnotetext{
${ }^{1}$ Also at the Institute of Theoretical and Mathematical Physics, MSU and Lebedev Institute, Moscow.
} 


\section{Contents}

1 Introduction 1

2 Renormalizability of $\lambda$-model: extended configuration space 5

2.1 Group space $\quad 7$

2.2 Coset space 8

2.32 -loop $\beta$-function of $\lambda$-model for group $G \quad 9$

2.42 -loop $\beta$-function of $\lambda$-model for symmetric space $G / H \quad 11$

2.5 Low-dimensional examples: $\lambda$-models for $\mathrm{SU}(2)$ and $\mathrm{SU}(2) / \mathrm{U}(1) \quad 13$

3 Renormalization of $\lambda$-model: standard configuration space 13

$\begin{array}{lll}3.1 \mathrm{SU}(2) / \mathrm{U}(1) & 14\end{array}$

$\begin{array}{lll}3.2 & \mathrm{SU}(2) & 15\end{array}$

3.2.1 $\mathrm{SU}(2) \times \mathrm{U}(1)$ invariant limit: abelian T-duality to squashed 3-sphere 17

$\begin{array}{lll}3.2 .2 & \text { Non-abelian dual of SU(2) PCM } & 19\end{array}$

4 Concluding remarks $\quad 20$

$\begin{array}{ll}\text { A Notation and conventions } & 21\end{array}$

B 2-loop $\beta$-function of squashed PCM and $G / H$ coset $\sigma$-models 22

\section{Introduction}

Certain $2 \mathrm{~d} \sigma$-models have the special property of renormalizablility, meaning they have only finitely many couplings running under RG flow. This property is expected to be closely connected with integrability [1-3]: the conservation of infinitely many hidden symmetry charges should reduce the RG flow in the infinite-dimensional space of $\sigma$-model couplings to a finite-dimensional one. Having previously been observed [1-6] only at the 1-loop (Ricci flow) level, it is important to study this reduction at higher loop orders to confirm its relation with integrability.

This question of higher loop orders was addressed recently in [7], where we showed that, starting from 2 loops, renormalizability requires a specific deformation of the classical target space geometry, which may be interpreted as the result of adding finite local counterterms.

In [7] we focused on the simplest examples of bosonic integrable $\sigma$-models with 2dimensional target spaces. Here we shall consider more general examples with higherdimensional target spaces and including B-field couplings. We shall concentrate on a particular class of integrable models: the $\lambda$-deformation based on a group $G$ or a symmetric 
space $G / H$ (related to the coset $\sigma$-model) $[8,9]$ with Lagrangian ${ }^{1}$

$$
\begin{aligned}
& \mathcal{L}=k\left(\mathcal{L}_{\mathrm{PCM}}(g)+\mathcal{L}_{\mathrm{WZ}}(g)\right. \\
&\left.\quad+\operatorname{Tr}\left[J_{+} A_{-}-A_{+} K_{-}+g^{-1} A_{+} g A_{-}-A_{+} A_{-}-\left(\lambda^{-1}-1\right) A_{+} P A_{-}\right]\right), \\
& \mathcal{L}_{\mathrm{PCM}}=-\frac{1}{2} \operatorname{Tr}\left[J_{+} J_{-}\right], \quad d B_{\mathrm{WZ}}=\frac{1}{6} \operatorname{Tr}[J \wedge J \wedge J], \quad J=g^{-1} d g, \quad K=d g g^{-1}, \\
& P= \begin{cases}1, & \text { group space } G \\
P_{G / H}, & \text { symmetric space } G / H,\end{cases}
\end{aligned}
$$

where $g \in G, A_{ \pm} \in \operatorname{Lie}(G)$ and $P_{G / H}$ is the projector onto the orthogonal complement of $\operatorname{Lie}(H)$ in $\operatorname{Lie}(G)$. Instead of $\lambda$ it is often convenient to use the parameters $\gamma$ or $\kappa$ defined as

$$
\gamma=\lambda^{-1}-1, \quad \kappa=\frac{1-\lambda}{1+\lambda} .
$$

This " $\lambda$-model" is special due to its close connection to the (gauged) WZW model

$$
\begin{aligned}
\mathcal{L}_{G}(g) & =\mathcal{L}_{\mathrm{PCM}}(g)+\mathcal{L}_{\mathrm{WZ}}(g), \\
\mathcal{L}_{G / H}(g, A) & =\mathcal{L}_{\mathrm{PCM}}(g)+\mathcal{L}_{\mathrm{WZ}}(g)+\operatorname{Tr}\left[J_{+} A_{-}-A_{+} K_{-}+g^{-1} A_{+} g A_{-}-A_{+} A_{-}\right] .
\end{aligned}
$$

For example, the $\lambda$-model for a group $G$ is a deformation of the $G / G$ gauged WZW model $\mathcal{L}_{G / G}(g, A)$ by the term $\gamma A_{+} A_{-}$. This model is a particular $H=G$ case of the one considered in [10-12]

$$
\mathcal{L}=k\left[\mathcal{L}_{G / H}(g, A)-\gamma \operatorname{Tr}\left(A_{+} A_{-}\right)\right], \quad g \in G, \quad A_{ \pm} \in \operatorname{Lie}(H) .
$$

This " $\gamma$-model" (1.6) interpolates between two conformal theories: $G / H$ gauged WZW model $(\gamma=0)$ and $G / H$ chiral gauged WZW model $(\gamma=-1)$ [13].

Let us note also that there is a $\mathbb{Z}_{2}$ transformation $[14,15]$ (see also $\left.[12,16]\right)^{2}$

$$
\begin{aligned}
& k \rightarrow-k, \quad \lambda \rightarrow \lambda^{-1}, \quad \text { i.e. } \kappa \rightarrow-\kappa, \\
& g \rightarrow g^{-1}, \quad A_{+} \rightarrow A_{+}-\left(1-\lambda^{-1}\right) P A_{+}, \quad A_{-} \rightarrow g A_{-} g^{-1}-K_{-},
\end{aligned}
$$

that maps the Lagrangian (1.2) to itself. The preservation of this symmetry at the quantum level may require a particular choice of regularization scheme (see below). Since the $\lambda \rightarrow$ 0 (or $\gamma \rightarrow \infty$ ) limit of the $\lambda$-model yields a (gauged) WZW model, we expect this to correspond to a fixed point of the RG flow. The transformation (1.7), (1.8) then implies that the same should apply to the limit $\lambda \rightarrow \infty$ (or $\gamma \rightarrow-1$ ). Indeed, in the group space case the $\lambda \rightarrow \infty$ limit of (1.2) is conformal: it is the $G / G$ chiral gauged WZW model, which, on integrating out $A_{ \pm}$, gives the $G$ WZW model at level $-k$. Similarly, in the coset case we find in this limit the $G / H$ gauged WZW model at level $-k$.

\footnotetext{
${ }^{1}$ Our notation and conventions are summarized in Appendix A. In particular, we use hermitian generators $T^{a}$ of the Lie algebra so that if $g=e^{v} \in G$ then $v=i T_{a} v^{a} \in \operatorname{Lie}(G)$ is anti-hermitian. The action is defined as $\mathcal{S}=\frac{1}{4 \pi} \int d^{2} \sigma \mathcal{L}$ so that $\mathcal{L}$ has extra factor of 2 compared to the "conventional" normalization.

${ }^{2}$ Such a symmetry was discussed in a similar $\sigma$-model context in [12] (see footnotes 3 and 6 there).
} 
Integrating out the $2 \mathrm{~d}$ gauge field $A_{ \pm}$in (1.2), i.e. reducing the model to the standard (or "physical") configuration space, one finds a $\sigma$-model with parameters $k$ and $\lambda$. The limit $\lambda \rightarrow 0$ yields the $G / H$ gauged WZW model (or $G$ WZW model in the group space case) with level $k$. As in the examples in [7], we shall find that to preserve renormalizability of this model at the 2-loop level with only the coupling $\lambda$ running, one must make a non-trivial modification of the classical target space geometry.

At the same time, our central observation will be that, before integrating out $A_{ \pm}$, the $\lambda$-model is renormalizable without any deformation. Changing the variables from $A_{ \pm}$to $h, \bar{h} \in G$ as $A_{+}=h \partial_{+} h^{-1}, A_{-}=\bar{h} \partial_{+} \bar{h}^{-1}$ gives a $\sigma$-model on the extended or "tripled" $(G \times G \times G)$ configuration space $(g, h, \bar{h})$. It may be interpreted as the sum of a decoupled $G$ WZW model and a deformation of the $G \times G$ WZW model by a particular left-right current interaction term. In the group space case, the form of the resulting action is then protected under the RG flow by the underlying chiral gauge symmetries together with the global symmetries.

For the coset $G / H$, the $\lambda$-model is formally defined for any choice of $H$ (with dependence on the choice of $H$ only through the projector $P$ in (1.2)). However, it is known to be integrable if $G / H$ is a symmetric space $[8,9] .{ }^{3}$ We shall find evidence that the model is also renormalizable if $G / H$ is a symmetric space, which is a further indication of a connection between integrability and renormalizability. ${ }^{4}$

The $\lambda$-model (1.2) admits a special limit $\lambda \rightarrow 1, k \rightarrow \infty$ with $\mathrm{h} \equiv 2 k(1-\lambda)$ fixed

$$
\begin{aligned}
& \lambda=1-\frac{1}{2} \mathrm{~h} k^{-1}+\mathcal{O}\left(k^{-2}\right), \quad k \rightarrow \infty, \\
& g=\exp \left(-\frac{1}{2} \mathrm{~h} k^{-1} v\right)=1-\frac{1}{2} \mathrm{~h} k^{-1} v+\mathcal{O}\left(k^{-2}\right),
\end{aligned}
$$

resulting in the following first-order Lagrangian [8]

$$
\mathcal{L}=\frac{1}{2} \mathrm{~h} \operatorname{Tr}\left[v\left(\partial_{+} A_{-}-\partial_{-} A_{+}+\left[A_{+}, A_{-}\right]\right)-A_{+} P A_{-}\right] .
$$

This is an interpolating model for non-abelian duality: integrating out $v$ in (1.10) gives the principal chiral model (PCM) on group $G$, or the $G / H$ symmetric space $\sigma$-model, with coupling $\sim \mathrm{h}^{-1}$, while integrating out $A_{ \pm}$gives the corresponding non-abelian dual (NAD) model. The renormalizability of the $\lambda$-model in the extended configuration space also applies in this limit: although the NAD of a group or symmetric space $\sigma$-model requires a non-trivial deformation at the 2-loop level [7], the interpolating model remains renormalizable without deformation. We conclude that, staying at the level of the interpolating model, non-abelian duality commutes with the RG flow beyond the 1-loop level (thus resolving problems discussed in [17-20]).

\footnotetext{
${ }^{3}$ In this case it is also related to the standard symmetric space $\sigma$-model (which is both integrable and renormalizable) in the NAD limit (1.9).

${ }^{4}$ For example, for more general cosets the symmetries may not be sufficient to rule out other possible current-current counterterms not present in the classical action.
} 
To study the 2-loop renormalizability of the above models we will be using the explicit expression for the $\beta$-function of the general bosonic $\sigma$-model

$$
\begin{aligned}
\mathcal{S} \equiv \frac{1}{4 \pi} \int d^{2} \sigma \mathcal{L} & =-\frac{1}{4 \pi} \int d^{2} \sigma\left(\eta^{r s} \mathrm{G}_{\mu \nu}(x)+\epsilon^{r s} \mathrm{~B}_{\mu \nu}(x)\right) \partial_{r} x^{\mu} \partial_{s} x^{\nu} \\
& =\frac{1}{4 \pi} \int d^{2} \sigma\left(\mathrm{G}_{\mu \nu}(x)+\mathrm{B}_{\mu \nu}(x)\right) \partial_{+} x^{\mu} \partial_{-} x^{\nu}
\end{aligned}
$$

In terms of the curvature $\hat{R}^{\mu}{ }_{\nu \rho \sigma}$ of the generalized connection $\hat{\Gamma}^{\mu}{ }_{\nu \rho}=\Gamma_{\nu \rho}^{\mu}(\mathrm{G})-\frac{1}{2} \mathrm{H}^{\mu}{ }_{\nu \rho}$ the 2-loop RG equation can be written as [21-26]

$$
\begin{aligned}
\frac{d\left(\mathrm{G}_{\mu \nu}+\mathrm{B}_{\mu \nu}\right)}{d t} & +L_{X}(\mathrm{G}+\mathrm{B})_{\mu \nu}+(d Y)_{\mu \nu}=\beta_{\mu \nu}^{(1)}+\beta_{\mu \nu}^{(2)}+\ldots \\
& =\hat{R}_{\mu \nu}+\frac{1}{2}\left[\hat{R}_{\nu}^{\rho \sigma \tau} \hat{R}_{\mu \rho \sigma \tau}-\frac{1}{2} \hat{R}^{\sigma \tau \rho}{ }_{\nu} \hat{R}_{\mu \rho \sigma \tau}+\frac{1}{2} \hat{R}_{\rho \mu \nu \sigma}\left(\mathrm{H}^{2}\right)^{\rho \sigma}\right]+\ldots
\end{aligned}
$$

Here $t$ is $\log$ of the RG mass scale, $L_{X}$ is the Lie derivative with respect to the vector $X$, corresponding to RG-dependent diffeomorphisms, and $d Y$ is an exact two-form, which is a total derivative when pulled back to the worldsheet. This 2-loop $\beta$-function is given in a particular "minimal" subtraction scheme $[23,24] .^{5}$

Let us now comment on the motivation behind the present work. In addition to understanding non-abelian duality beyond the 1-loop level, investigating the $\lambda$-model and its quantum corrections is of more general interest in the context of integrable deformations of superstring actions in special AdS-type backgrounds. Integrability has been a powerful tool in the proposed solution of the spectral problem for string theory in on $\operatorname{Ad} S_{5} \times S^{5}$ dual to the large- $N$ maximally supersymmetric YM theory $[27,28]$. This motivates the study of further similar models, potentially leading to new exact solutions of strings in curved spaces and dual gauge theories. By now there are many examples, including those based on lower-dimensional AdS spaces [29, 30], as well as deformed backgrounds, such as the well-studied $\beta$-deformation [31-33].

The $\lambda$-deformation of the $A d S_{5} \times S^{5}$ superstring [34] belongs to a more general class of integrable deformations not obtained by T-duality. It is a deformation of the nonabelian dual model of the undeformed superstring model and is closely related to the $\eta$-deformation [35], which is a deformation of the superstring action itself. The latter generalises the bosonic $\eta$-model of $[36,37]$. More precisely, the $\lambda$-model and $\eta$-model are related by the Poisson-Lie duality $[38,39]$ (which is a generalisation of non-abelian duality) and a particular analytic continuation [15, 40-43]. While both models describe a string propagating in a type II supergravity background [44, 45], much remains to be understood about their structure. Probing the quantum properties of the bosonic $\eta$-model and $\lambda$-model (even though they are not themselves scale-invariant theories suitable for defining string models) can provide valuable insights into their superstring counterparts. For example,

\footnotetext{
${ }^{5}$ Alternative "minimal" schemes are related to this one by $(\mathrm{G}+\mathrm{B})_{\mu \nu} \rightarrow(\mathrm{G}+\mathrm{B})_{\mu \nu}+a_{1} R_{\mu \nu}+a_{2}\left(\mathrm{H}^{2}\right)_{\mu \nu}+$ $a_{3} \hat{R}_{\mu \nu}$. Since $\beta_{\mu \nu}^{(1)}=\hat{R}_{\mu \nu}$ it follows that shifts by $\hat{R}_{\mu \nu}$ will leave $\beta_{\mu \nu}^{(2)}$ invariant. On the other hand, shifts by $R_{\mu \nu}$ and $\left(\mathrm{H}^{2}\right)_{\mu \nu}$ do modify $\beta_{\mu \nu}^{(2)}$, and hence, in the case of non-trivial B-field, the 2-loop RG equation is no longer scheme-independent [23, 24]. One other scheme that will be useful in our discussion of T-duality in section 3 is related to the minimal one in (1.12) by $(\mathrm{G}+\mathrm{B})_{\mu \nu} \rightarrow(\mathrm{G}+\mathrm{B})_{\mu \nu}+\frac{1}{4}\left(\mathrm{H}^{2}\right)_{\mu \nu}$.
} 
the relation via the Poisson-Lie duality was first understood in the bosonic case. It is thus natural to first explore the question of quantum corrections by studying the bosonic models.

It is also worth emphasizing that the bosonic models are of interest in their own right in the context of investigation of general integrable $2 \mathrm{~d}$ theories. The $\eta$-model has played an important role in generalizing the duality between the deformed $O(3)$ and $O(4)$ sigma models and massive integrable QFTs [1-3] to higher-rank groups [4-6]. While the dual theories are quantum-exact, the $\sigma$-model side of the duality is only understood so far to leading order in the loop expansion. Therefore, after finding quantum corrections to integrable $\sigma$-models consistent with renormalizability, studying their compatibility with this duality may be a useful way to further explore the conjectured relationship between integrability and renormalizability.

The rest of the paper is organized as follows. In section 2 we consider the $\lambda$-model on the extended configuration space $\left(g, A_{+}, A_{-}\right)$. We argue that it should be renormalizable with only the parameter $\lambda$ running and compute the 2-loop $\beta$-function of the $\lambda$-model based on general groups and symmetric spaces. As a consequence, the same renormalizability conclusion holds for the model (1.10) interpolating between the PCM and its NAD, with the same 2-loop $\beta$-function for h as in the PCM (and the same in the symmetric space case).

In section 3 we study the renormalization of the $\lambda$-model defined by the standard $\sigma$ model action, after integrating out $A_{ \pm}$. In this case its invariance under the 2-loop RG flow requires a specific deformation of the classical geometry. While in the $\mathrm{SU}(2) / \mathrm{U}(1)$ case the required counterterm is simple [7], in the $\mathrm{SU}(2)$ case, the corresponding quantumcorrected $\sigma$-model action has a rather intricate structure. We also consider a particular limit of the $\mathrm{SU}(2) \lambda$-model where it becomes T-dual to a $\sigma$-model for a squashed 3 -sphere, explaining the consistency of the quantum deformation of the original $\lambda$-model with the known quantum correction to the T-duality transformation rule. We also discuss the 2-loop $\beta$-function for the NAD of the SU(2) PCM.

Some concluding remarks are made in section 4. In appendix A we summarize our notation and group-theory conventions. In appendix B we compute the 2 -loop $\beta$-function for a two-coupling "squashed" principal chiral model that interpolates between the $G$ group space PCM and the $G / H$ coset $\sigma$-model, determining also the 2-loop $\beta$-function for the latter.

\section{Renormalizability of $\lambda$-model: extended configuration space}

In this section we shall study renormalization of the $\lambda$-model on the extended configuration space. It is first useful to draw an analogy with the $\gamma$-model $[10,11]$ defined in (1.6) (where $G$ and $H \subset G$ are simple Lie groups) that interpolates between the gauged WZW (gWZW) and chiral gauged WZW (cWZW) theories. Changing variables $\left(A_{+}, A_{-}\right) \rightarrow(h, \bar{h})$ as

$$
A_{+}=h \partial_{+} h^{-1}, \quad A_{-}=\bar{h} \partial_{-} \bar{h}^{-1}, \quad h, \bar{h} \in H,
$$


and using the Polyakov-Wiegmann identity [46], we can rewrite the Lagrangian in (1.6) as a combination of WZW models (cf. (1.5))

$$
\begin{aligned}
\mathcal{L} & =k\left(\mathcal{L}_{G / H}(g, A)-\gamma \operatorname{Tr}\left[A_{+} A_{-}\right]\right) \\
& =k\left(\mathcal{L}_{G}\left(h^{-1} g \bar{h}\right)-\mathcal{L}_{H}\left(h^{-1} \bar{h}\right)-\gamma\left[\mathcal{L}_{H}\left(h^{-1} \bar{h}\right)-\mathcal{L}_{H}\left(h^{-1}\right)-\mathcal{L}_{H}(\bar{h})\right]\right) .
\end{aligned}
$$

The change of variables in (2.1) results in a Jacobian contributing to the action as [46, 47]

$$
\begin{aligned}
\Delta \mathcal{L} & =-2 c_{H}\left(\mathcal{L}_{H}\left(h^{-1} \bar{h}\right)+\mathrm{q} \operatorname{Tr}\left[A_{+} A_{-}\right]\right) \\
& =-2 c_{H}\left(\mathcal{L}_{H}\left(h^{-1}\right)+\mathcal{L}_{H}(\bar{h})+(\mathrm{q}-1) \operatorname{Tr}\left[\partial_{+} h h^{-1} \partial_{-} \bar{h} \bar{h}^{-1}\right]\right),
\end{aligned}
$$

where $c_{H}=c_{2}(H)$ is the dual Coxeter number of $H$ and an arbitrary coefficient $\mathrm{q}$ parametrizes the ambiguity of adding a local counterterm $\operatorname{Tr}\left[A_{+} A_{-}\right]$. Combining (2.3) and the classical Lagrangian (2.2) gives

$$
\mathcal{L}=k \mathcal{L}_{G}\left(h^{-1} g \bar{h}\right)-\left(k+2 c_{H}\right) \mathcal{L}_{H}\left(h^{-1} \bar{h}\right)-\left(k \gamma+2 \mathrm{q} c_{H}\right)\left[\mathcal{L}_{H}\left(h^{-1} \bar{h}\right)-\mathcal{L}_{H}\left(h^{-1}\right)-\mathcal{L}_{H}(\bar{h})\right] .
$$

In the special cases $\gamma=0$ (gWZW model) and $\gamma=-1$ (cWZW model), we can choose $\mathrm{q}$ such that (2.4) is a sum of WZW models $[10,11]$

$$
\begin{array}{llll}
\text { gWZW : } & \gamma=0, & \mathrm{q}=0, & \mathcal{L}=k \mathcal{L}_{G}\left(h^{-1} g \bar{h}\right)-\left(k+2 c_{H}\right) \mathcal{L}_{H}\left(h^{-1} \bar{h}\right), \\
\text { cWZW : } & \gamma=-1, & \mathrm{q}=-1, & \mathcal{L}=k \mathcal{L}_{G}\left(h^{-1} g \bar{h}\right)-\left(k+2 c_{H}\right)\left[\mathcal{L}_{H}\left(h^{-1}\right)+\mathcal{L}_{H}(\bar{h})\right] .
\end{array}
$$

Since the arguments $\left\{\tilde{g}=h^{-1} g \bar{h}, \tilde{h}=h^{-1} \bar{h}\right\}$ in (2.5) or $\left\{\tilde{g}=h^{-1} g \bar{h}, h^{-1}, \bar{h}\right\}$ in (2.6) may be treated as independent fields, it follows that these two models are conformally invariant.

For general values of $\gamma$, choosing $\mathrm{q}=-1$ as in the cWZW case (2.6), we may rewrite $(2.4)$ as

$$
\begin{aligned}
\mathcal{L} & =k \mathcal{L}_{G}(\tilde{g})+\mathcal{L}^{\prime}(\hat{h}, \bar{h}), \quad \hat{g} \equiv h^{-1} g \bar{h} \in G, \quad \hat{h} \equiv h^{-1} \in H, \\
\mathcal{L}^{\prime} & =-\left(k+2 c_{H}\right)\left[\mathcal{L}_{H}(\hat{h})+\mathcal{L}_{H}(\bar{h})\right]+k(1+\gamma) \operatorname{Tr}\left[\hat{h}^{-1} \partial_{+} \hat{h} \partial_{-} \bar{h} \bar{h}^{-1}\right] .
\end{aligned}
$$

This model is defined on the extended configuration space $(\tilde{g}, \hat{h}, \bar{h}) \in G \times H \times H$. The first $G$ WZW term (which is conformal on its own) decouples and then we are left with the "truncated" model $\mathcal{L}^{\prime}$ on $H \times H$ which is simply a sum of two group $H$ WZW models perturbed by the product of the left and right currents. Like the chiral gauged WZW model (2.6) the Lagrangian $\mathcal{L}^{\prime}$ in (2.8) is invariant under the chiral gauge symmetry $\hat{h} \rightarrow$ $u\left(\sigma^{-}\right) \hat{h}, \bar{h} \rightarrow \bar{h} w\left(\sigma^{+}\right), u, w \in H$ as well as the global $H$ symmetry $\hat{h} \rightarrow \hat{h} v_{0}, \bar{h} \rightarrow$ $v_{0}^{-1} \bar{h}, v_{0} \in H$. As we shall argue for the $\lambda$-model, these two symmetries imply that the $\gamma$-model is also renormalizable with only one coupling $\gamma$ running.

Let us note that the $\gamma$-model (1.6), (2.2) admits also a generalization similar to the coset case of the $\lambda$-model in (1.2): with $\gamma A_{+} A_{-}$term replaced by $\gamma A_{+} P A_{-}$, where $P$ is the projector onto the $H / F$ coset part of the algebra of $H$ (with $F \subset H \subset G$ ). When $H / F$ is a symmetric space this model should again be renormalizable on the extended configuration space $(\tilde{g}, \hat{h}, \bar{h}) \in G \times H \times H$ due to chiral gauge symmetry. 


\subsection{Group space}

Let us now apply similar arguments to the $\lambda$-model for the group $G$, which is given by (1.2) with $P=1$. Taking $H=G$ in (2.1), so that now $h, \bar{h} \in G$, we obtain (2.4) with $H=G$ and $c_{H} \rightarrow c_{G}=c_{2}(G)$. It will represent the $\lambda$-model as a $\sigma$-model on a "tripled" configuration space $\left(\tilde{g}=h^{-1} g \bar{h}, h^{-1}, \bar{h}\right) \in G \times G \times G$. Since the q-dependent term in (2.3) is simply equivalent to a finite quantum (order $1 / k$ ) redefinition $\gamma \rightarrow \gamma+\frac{2 c_{G}}{k} \mathrm{q}$ of the parameter $\gamma$, we are free to fix $q$ to a specific value, $q=-1$, as in the cWZW case (2.6) and in (2.7), (2.8). This gives the following analog of $(2.7)$

$$
\begin{aligned}
\mathcal{L} & =k \mathcal{L}_{G}(\tilde{g})-k(1+\gamma) \mathcal{L}_{G}\left(h^{-1} \bar{h}\right)+\left(k \gamma-2 c_{G}\right)\left[\mathcal{L}_{G}\left(h^{-1}\right)+\mathcal{L}_{G}(\bar{h})\right] \\
& =k \mathcal{L}_{G}(\tilde{g})-\left(k+2 c_{G}\right)\left[\mathcal{L}_{G}\left(h^{-1}\right)+\mathcal{L}_{G}(\bar{h})\right]-k(1+\gamma) \operatorname{Tr}\left[\partial_{+} h h^{-1} \partial_{-} \bar{h} \bar{h}^{-1}\right] .
\end{aligned}
$$

We thus obtain the same tripled theory as (2.7), (2.8), now with $H \rightarrow G$ : the first term is the $G$ WZW model for $\tilde{g}$, which decouples from the $(\hat{h}, \bar{h})$ theory described by the "truncated" Lagrangian

$$
\begin{aligned}
\mathcal{L}^{\prime}(\hat{h}, \bar{h}) & =-\tilde{k}\left(\mathcal{L}_{G}(\hat{h})+\mathcal{L}_{G}(\bar{h})-\tilde{\lambda} \operatorname{Tr}\left[\hat{h}^{-1} \partial_{+} \hat{h} \partial_{-} \bar{h} \bar{h}^{-1}\right]\right), \quad \hat{h} \equiv h^{-1}, \bar{h} \in G, \\
\tilde{k} & \equiv k+2 c_{G}, \quad \tilde{\lambda} \equiv \frac{k}{k+2 c_{G}}(1+\gamma)=\frac{k}{\tilde{k}} \lambda^{-1}=\lambda^{-1}+\mathcal{O}\left(k^{-1}\right) .
\end{aligned}
$$

$\mathcal{L}^{\prime}$ may be interpreted as the Lagrangian for the two WZW models for the two groups $G$ with the same level $-\left(k+2 c_{G}\right)$ perturbed by a product of the left current of one group and the right current of the other. ${ }^{6}$

Our central observation is that the model (2.10) is renormalizable with only the coupling $\lambda$ (or $\tilde{\lambda}$ ) running with the RG scale ( $k$ should not run as it appears as the coefficient of the WZ term). Indeed, the structure of (2.10) is protected by the same chiral gauge symmetry present in the cWZW model (2.6) and in the $\gamma$-model (2.8),

$$
\hat{h} \rightarrow u\left(\sigma^{-}\right) \hat{h}, \quad \bar{h} \rightarrow \bar{h} w\left(\sigma^{+}\right), \quad u, w \in G .
$$

This symmetry, together with the global $G$ symmetry

$$
\hat{h} \rightarrow \hat{h} g_{0}, \quad \bar{h} \rightarrow g_{0}^{-1} \bar{h}, \quad g_{0} \in G,
$$

prohibits the appearance of other current-current interaction terms under the RG flow. ${ }^{7}$

It is then straightforward to compute the $\beta$-function for $\lambda$ in the large $k$ perturbation theory, which we will do in section 2.3. The two fixed points of the RG flow for (2.10) will be $\tilde{\lambda}=\infty, 0$, corresponding to $\lambda=0, \infty$ respectively. $^{8}$

\footnotetext{
${ }^{6}$ Similar models were discussed in the past, e.g., in [48-50]. A generalization of this model was also considered in [51-54], where it was interpreted as a special case of a "doubly $\lambda$-deformed" $\sigma$-model. Our path-integral relation between the $\lambda$-model (1.2) and the truncated model (2.10) should be equivalent (at least at the classical and 1-loop level) to the canonical equivalence between the doubly $\lambda$-deformed model and two copies of the $\lambda$-model found in [53] (upon setting one of the two $\lambda$-parameters to zero). The leading order in $1 / k$ (1-loop) renormalization of similar models was studied earlier in $[55,56]$.

${ }^{7}$ The presence of this symmetry is also a manifestation of the integrability of the original $\lambda$-model.

${ }^{8}$ Note that for general values of $\mathrm{q}$ in $(2.3),(2.4)$ one gets $\tilde{k} \tilde{\lambda}=k \lambda^{-1}+2(1+\mathrm{q}) c_{G}$ in (2.11). Finite redefinitions of parameters like $k \rightarrow \tilde{k}$ and $\lambda \rightarrow \tilde{\lambda}$ in (2.11) are not important for the discussion of renormalization in $1 / k$ perturbation theory, simply reflecting the freedom of scheme choice. They may, however, correct the 1-loop fixed points $\lambda=0, \infty$ of the RG flow.
} 


\subsection{Coset space}

Next, let us consider the $\lambda$-model for the coset $G / H$, i.e. setting $P=P_{G / H}$ in (1.2). Repeating the same steps, i.e. using (2.1) to introduce $h, \bar{h} \in G$, including the contribution from the Jacobian (2.3), setting $\tilde{g}=h^{-1} g \bar{h}$ and $\hat{h}=h^{-1}$, we get

$$
\mathcal{L}=k \mathcal{L}_{G}(\tilde{g})-\left(k+2 c_{G}\right) \mathcal{L}_{G}(\hat{h} \bar{h})+k \gamma \operatorname{Tr}\left[\hat{h}^{-1} \partial_{+} \hat{h} P \partial_{-} \bar{h} \bar{h}^{-1}\right]+2 \mathrm{q} c_{G} \operatorname{Tr}\left[\hat{h}^{-1} \partial_{+} \hat{h} \partial_{-} \bar{h} \bar{h}^{-1}\right]
$$

Classically (i.e. for large $k$ ) this model has the expected $H$ gauge symmetry

$$
\hat{h} \rightarrow \hat{h} f, \quad \bar{h} \rightarrow f^{-1} \bar{h}, \quad f=f\left(\sigma^{+}, \sigma^{-}\right) \in H .
$$

To preserve this gauge symmetry in (2.14) let us choose (as in the gWZW case (2.5)) q $=0$, thus obtaining

$$
\begin{aligned}
\mathcal{L} & =k \mathcal{L}_{G}(\tilde{g})-\left(k+2 c_{G}\right) \mathcal{L}_{G}(\hat{h} \bar{h})+k \gamma \operatorname{Tr}\left[\hat{h}^{-1} \partial_{+} \hat{h} P \partial_{-} \bar{h} \bar{h}^{-1}\right] \\
& =k \mathcal{L}_{G}(\tilde{g})-\left(k+2 c_{G}\right)\left[\mathcal{L}_{G}(\hat{h})+\mathcal{L}_{G}(\bar{h})\right]+\operatorname{Tr}\left[\hat{h}^{-1} \partial_{+} \hat{h}\left(k+2 c_{G}+k \gamma P\right) \partial_{-} \bar{h} \bar{h}^{-1}\right]
\end{aligned}
$$

As in (2.9) the WZW term for the field $\tilde{g}$ decouples, leaving us with

$$
\begin{aligned}
\mathcal{L}^{\prime}(\hat{h}, \bar{h}) & =-\tilde{k}\left(\mathcal{L}_{G}(\hat{h} \bar{h})-(\tilde{\lambda}-1) \operatorname{Tr}\left[\hat{h}^{-1} \partial_{+} \hat{h} P \partial_{-} \bar{h} \bar{h}^{-1}\right]\right) \\
& =-\tilde{k}\left(\mathcal{L}_{G}(\hat{h})+\mathcal{L}_{G}(\bar{h})-\operatorname{Tr}\left[\hat{h}^{-1} \partial_{+} \hat{h}(1-P) \partial_{-} \bar{h} \bar{h}^{-1}\right]-\tilde{\lambda} \operatorname{Tr}\left[\hat{h}^{-1} \partial_{+} \hat{h} P \partial_{-} \bar{h} \bar{h}^{-1}\right]\right), \\
\tilde{k} & \equiv k+2 c_{G}, \quad \tilde{\lambda} \equiv 1+\frac{k \gamma}{k+2 c_{G}}=\frac{k}{\tilde{k}} \lambda^{-1}+\frac{2 c_{G}}{\tilde{k}}=\lambda^{-1}+\mathcal{O}\left(k^{-1}\right) .
\end{aligned}
$$

$\mathcal{L}^{\prime}$ represents the $G \times G$ WZW model deformed by the product of the left and right currents projected to the subgroup $P_{H}=1-P$ and the coset $P_{G / H}=P$. While it is again invariant under the chiral transformations (2.12), here it is not immediately clear that this theory is renormalizable with only one coupling running: in principle, different gauge-invariant projections of the product of currents may appear as independent counterterms. When $G / H$ is an irreducible symmetric space (which also implies the integrability of the $\lambda$ model), the coset part of the algebra of $G$ transforms in an irreducible representation of Lie $(H)$. Thus the model is renormalizable with only $\lambda$ running since new current-current interaction terms are prohibited by symmetries. ${ }^{9}$ We shall see this explicitly at the 2-loop level in section 2.4 below.

In this case the two expected fixed points of the RG flow for $(2.17), \tilde{\lambda}^{-1}=0$ and $\tilde{\lambda}^{-1}=\infty$, now correspond to $\lambda=0$ and $\lambda=-\frac{k}{2 c_{G}}$ due to the shift in (2.18). That is, one of the 1-loop fixed points, $\lambda=\infty$, is corrected.

\footnotetext{
${ }^{9}$ If the coset directions $G / H$ transform in an irreducible representation of $\operatorname{Lie}(H)$ that is reducible over $\mathbb{C}$, i.e. decomposes into two complex conjugate representations, then there can be additional real gaugeinvariant terms that respect the chiral symmetry. An example would be $i \operatorname{Tr}\left[\hat{h}^{-1} \partial_{+} \hat{h}\left(P_{+}-P_{-}\right) \partial_{-} \bar{h} \bar{h}^{-1}\right]$ where $P=P_{+}+P_{-}$and $P_{ \pm}$are projectors onto the conjugate representations. Such a term should not be generated under the RG flow as it is not invariant under $\hat{h} \leftrightarrow \bar{h}^{-1}$ plus parity, which is a symmetry of (2.17). More generally, we expect any new terms to be excluded by symmetries.
} 


\section{$2.3 \quad 2$-loop $\beta$-function of $\lambda$-model for group $G$}

Let us now compute the 2-loop $\beta$-function for the model (2.10) in the case that $G$ is a compact simple group, explicitly demonstrating its renormalizability with only one parameter $\lambda$ running (the 1-loop $\beta$-function for this $G \times G$ model was computed in $[52,54]$ ). ${ }^{10} \mathrm{We}$ shall use large $k$ perturbation theory with $\lambda$ arbitrary.

Let us introduce the basis $\left\{T^{a}\right\}$ for $\operatorname{Lie}(G)$ (see appendix A for conventions). In a slight abuse of notation, we shall use the indices $a$ and $\bar{a}$ for the two $G$-valued fields $\hat{h}$ and $\bar{h}$ in (2.10) respectively (with the tangent space index for $G \times G$ denoted as $A=\{a, \bar{a}\}$ ). As in [54] we introduce the vielbein

$$
\begin{aligned}
& E^{A}=\left(e^{a}, e^{\bar{a}}\right)=\left(\sqrt{1-\tilde{\lambda}^{2}} J^{a}, K^{\bar{a}}+\tilde{\lambda} J^{a}\right), \\
& J^{a} \equiv \operatorname{Tr}\left[T^{a} \hat{h}^{-1} d \hat{h}\right], \quad K^{\bar{a}} \equiv \operatorname{Tr}\left[T^{a} d \bar{h} \bar{h}^{-1}\right],
\end{aligned}
$$

where $J^{a}$ and $K^{a}$ are the currents that appear in the deformation term in (2.10). Up to permutations, the non-zero components of the metric and H-tensor of the $G \times G$ model (2.10) are (cf. $(1.11))^{11}$

$$
\begin{aligned}
\mathrm{G}_{a b} & =\mathrm{G}_{\bar{a} \bar{b}}=\frac{\tilde{k}}{2} \delta_{a b}, \\
\mathrm{H}_{a b c} & =-\frac{\tilde{k}}{2} \frac{\sqrt{1-\tilde{\lambda}^{2}}(1+2 \tilde{\lambda})}{(1+\tilde{\lambda})^{2}} f_{a b c}, \quad \mathrm{H}_{\bar{a} \bar{b} \bar{c}}=-\frac{\tilde{k}}{2} f_{a b c}, \quad \mathrm{H}_{\bar{a} b c}=-\frac{\tilde{k}}{2} \frac{\tilde{\lambda}}{1+\tilde{\lambda}} f_{a b c} .
\end{aligned}
$$

Our aim is to compute the corresponding $\beta$-function in (1.12). Let us formally define the torsion as $T^{A}=\frac{1}{2} \mathrm{H}^{A}{ }_{B C} E^{B} \wedge E^{C}$ where the tangent space index is raised with the inverse of the metric (2.21). Then from the Cartan structure equation $d E^{A}+\hat{\omega}^{A}{ }_{B} \wedge E^{B}=T^{A}$, we obtain the torsionful spin connection $\hat{\omega}_{B}^{A}=\hat{\omega}^{A}{ }_{B C} E^{C}$ with non-zero components

$$
\left(\tilde{\lambda}^{-1}+1\right) \hat{\omega}_{b}^{a}=\hat{\omega}_{\bar{b}}^{\bar{a}_{\bar{b}}}=f_{b c}^{a}\left(-\frac{\tilde{\lambda}}{\sqrt{1-\tilde{\lambda}^{2}}} e^{c}+e^{\bar{c}}\right) .
$$

The non-zero components of the curvature, $\hat{R}_{B}^{A}=d \hat{\omega}^{A}{ }_{B}+\hat{\omega}^{A}{ }_{C} \wedge \hat{\omega}^{C}{ }_{B}=\frac{1}{2} \hat{R}_{B C D}^{A} E^{C} \wedge E^{D}$, are then found to be

$$
\left(\tilde{\lambda}^{-2}-1\right) \hat{R}_{b d e}^{a}=-\sqrt{\tilde{\lambda}^{-2}-1} \hat{R}_{b d \bar{e}}^{a}=\hat{R}_{b \bar{d} \bar{e}}^{a}=\frac{\tilde{\lambda}}{(1+\tilde{\lambda})^{2}} f_{b c}^{a} f_{d e}^{c} .
$$

Plugging (2.24) into (1.12) one obtains

$$
\left(\beta_{\mu \nu}^{(1)}+\beta_{\mu \nu}^{(2)}\right) d x^{\mu} \otimes d x^{\nu}=\sum_{a=\bar{a}=1}^{\operatorname{dim} G}\left[\frac{2 c_{G} \tilde{\lambda}^{2}}{(1+\tilde{\lambda})^{2}}+\frac{4 c_{G}^{2}}{\tilde{k}} \frac{\tilde{\lambda}^{4}(1-2 \tilde{\lambda})}{(1+\tilde{\lambda})^{5}(1-\tilde{\lambda})}\right] J^{a} \otimes K^{\bar{a}} .
$$

\footnotetext{
${ }^{10}$ On the standard configuration space the 1-loop $\beta$-function of the $\lambda$-model for group space $G$ or symmetric space $G / H$ can be extracted from [12] and was also explicitly computed in [57, 58].

${ }^{11}$ Note that in our conventions (with hermitian generators $T^{a}$, see Appendix A) the vielbein defined in (2.19) and the components $\mathrm{H}_{A B C} \sim f_{A B C}$ in (2.22) are imaginary but the 3-form $\mathrm{H}=\frac{1}{6} \mathrm{H}_{A B C} E^{A} \wedge E^{B} \wedge E^{C}$ is real.
} 
Here $\otimes$ indicates that the product is not symmetrized. We conclude that only the $\tilde{\lambda}$ dependent term in (2.10) gets renormalized, i.e. the 2-loop RG equation (1.12) is solved with $X^{\mu}=Y_{\mu}=0$ and

$$
\frac{d}{d t} \tilde{k}=0, \quad \frac{d}{d t} \tilde{\lambda}=\frac{2 c_{G}}{\tilde{k}}\left[\frac{\tilde{\lambda}^{2}}{(1+\tilde{\lambda})^{2}}+\frac{2 c_{G}}{\tilde{k}} \frac{\tilde{\lambda}^{4}(1-2 \tilde{\lambda})}{(1+\tilde{\lambda})^{5}(1-\tilde{\lambda})}\right] .
$$

Here the 1-loop term agrees with $[57,58]$ (recall that $\tilde{\lambda}=\lambda^{-1}+\ldots$ and $\tilde{k}=k+\ldots$, cf. (2.18)). Note also that, while the Lagrangian (2.10) is linear in $\tilde{\lambda}$, the non-polynomiality of (2.26) in $\tilde{\lambda}$ is a direct consequence of the exactness of the $\sigma$-model $\beta$-function (1.12) in the metric $\mathrm{G}$.

The level $k$ is thus RG-invariant as it should be and, as expected, $\tilde{\lambda}=\infty, 0$ are fixed points of the RG flow. Expressing $\tilde{k}$ and $\tilde{\lambda}$ in (2.11) in terms of $k$ and the coupling $\kappa$ using (1.4), we find

$$
\frac{d}{d t} k=0, \quad \frac{d}{d t} \kappa=\frac{c_{G}}{4 k}\left(1-\kappa^{2}\right)^{2}\left[1+\frac{c_{G}(1-\kappa)^{2}\left(1-10 \kappa-3 \kappa^{2}\right)}{8 k \kappa}\right] .
$$

At the fixed point $\kappa=1$ (equivalent to $\lambda=0$ or $\tilde{\lambda}=\infty$ ), the $\lambda$-model reduces to the $G$ WZW model with level $k$. The other fixed point $\kappa=-1$ (equivalent to $\lambda=\infty$ or $\tilde{\lambda}=0)$ is the $G / G$ cWZW model, which reduces to the $G$ WZW model with level $-k$ after integrating out the gauge field. ${ }^{12,13}$

Note that the 2-loop term in (2.27) is scheme-dependent: it can be changed by redefining $\kappa$ by a $1 / k$ term (or shifting $k$ by a finite term). ${ }^{14}$ Even though $k$ is not running, we effectively have a 2 -coupling theory (with $1 / k$ playing the role of a loop-counting parameter) so only the 1-loop term in the $\beta$-function (2.27) is scheme-independent.

In a general scheme, the symmetry $k \rightarrow-k, \lambda \rightarrow \lambda^{-1}$ (1.7) of the 1-loop RG equation in $(2.26),(2.27)$ is not manifest at the 2-loop level. To preserve it requires a particular formulation of the quantum theory, i.e. a specific definition of the couplings, or choice of scheme. For example, if we redefine the parameters as

$$
(\tilde{k}, \tilde{\lambda}) \rightarrow(\mathrm{k}, \bar{\lambda}), \quad \tilde{k}=\mathrm{k}+c_{G}, \quad \tilde{\lambda}=\bar{\lambda}\left[1+c_{G} \mathrm{k}^{-1} \frac{1-\bar{\lambda}}{1+\bar{\lambda}}\right],
$$

then the RG equation for $\bar{\lambda}$ resulting from (2.26) is

$$
\frac{d}{d t} \bar{\lambda}=\frac{2 c_{G}}{\mathrm{k}} \frac{\bar{\lambda}^{2}}{(1+\bar{\lambda})^{2}}\left[1-\frac{2 c_{G}}{\mathrm{k}} \frac{\bar{\lambda}^{2}}{(1+\bar{\lambda})^{2}} \frac{\bar{\lambda}+\bar{\lambda}^{-1}-1}{1-\bar{\lambda}^{2}}\right]
$$

which is invariant under the following quantum version of (1.7)

$$
\mathrm{k} \rightarrow-\mathrm{k}, \quad \bar{\lambda} \rightarrow \bar{\lambda}^{-1}
$$

\footnotetext{
${ }^{12}$ To compare, for the PCM model plus WZ term $\mathcal{L}=\mathrm{h} \mathcal{L}_{\mathrm{PCM}}+k \mathcal{L}_{\mathrm{WZ}}$ (which becomes the WZW model for $\left.\varkappa \equiv \frac{\mathrm{h}}{k}=1\right)$ the 2-loop RG equation for $\varkappa[23,24,59,60]$ is $\frac{d}{d t} \varkappa=\frac{c_{G}}{k}\left(1-\varkappa^{-2}\right)\left[1+\frac{c_{G}}{2 k \varkappa}\left(1-3 \varkappa^{-2}\right)\right]$.

${ }^{13}$ Taking $\kappa=0$ or $\lambda=1$ with finite $k$ (in contrast to the NAD limit (1.9)) gives the $G / G$ gWZW model in which one can fix a gauge so that the remaining degrees of freedom correspond to the Cartan torus.

${ }^{14}$ For example, the 2-loop term in (2.26) will depend on the parameter q in (2.3), (2.4), cf. footnote 8.
} 
Note that here $\mathrm{k}=k+c_{G}$ is the same as the shifted level of the WZW theory. It is also worth observing that (2.28) is not the only redefinition that restores the symmetry (1.7). Indeed, even restricting to those that preserve the existence of the fixed points and the NAD limit discussed below, there are many.

In the NAD limit (1.9) (i.e. $\kappa=\frac{1}{4} \mathrm{~h} k^{-1}, k \rightarrow \infty$ ) the 2-loop RG equation (2.27) becomes

$$
\frac{d}{d t} \mathrm{~h}=c_{G}+\frac{1}{2} c_{G}^{2} \mathrm{~h}^{-1}
$$

Here the 2-loop term is scheme-independent since this is now a 1-coupling theory. As verified in appendix $\mathrm{B}$, the $\beta$-function (2.31) matches the standard expression (see, e.g., [21, $61,62]$ and footnote 12) for the 2-loop $\beta$-function of the PCM on a compact simple group $G$ (with the coupling $\mathrm{h}=2 \mathrm{~g}^{-2}$ ). As in the $\mathrm{SU}(2) / \mathrm{U}(1)$ example discussed in [7], this demonstrates that the NAD of the PCM has the same 2-loop $\beta$-function as the PCM itself, extending the previous conclusions $[63,64]$ on the 1-loop quantum equivalence of models related by the non-abelian duality to the 2-loop level.

\subsection{2 -loop $\beta$-function of $\lambda$-model for symmetric space $G / H$}

We now turn to the case of the $\lambda$-model based on the compact irreducible symmetric space $G / H$, explicitly demonstrating its renormalizability by computing the 2 -loop $\beta$-function for the model (2.17). The computation runs analogously to the group space case discussed in section 2.3. We decompose the basis $\left\{T^{a}\right\}$ of $\operatorname{Lie}(G)$ according to the $\mathbb{Z}_{2}$ grading of the Lie algebra with $\left\{T^{\alpha}\right\}$ spanning $\operatorname{Lie}(H)$ and $\left\{T^{i}\right\}$ its complement (cf. appendix A). ${ }^{15}$ The tangent space index for $G \times G$ now splits as $A=\{a ; \bar{a}\}=\{\alpha, i ; \bar{\alpha}, \bar{l}\}$, with the unbarred and barred indices corresponding to the two $G$-valued fields $\hat{h}$ and $\bar{h}$.

Here we use the vielbein

$$
E^{A}=\left(e^{i}, e^{\bar{\imath}}, e^{\alpha}, e^{\bar{\alpha}}\right)=\left(\sqrt{1-\tilde{\lambda}^{2}} J^{i}, K^{\bar{\imath}}+\tilde{\lambda} J^{i}, J^{\alpha}+K^{\bar{\alpha}}, J^{\alpha}-K^{\bar{\alpha}}\right) .
$$

Up to permutations, the non-zero components of the metric and H-tensor are

$$
\begin{aligned}
& \mathrm{G}_{i j}=\mathrm{G}_{\bar{\imath} \bar{\jmath}}=\frac{\tilde{k}}{2} \delta_{i j}, \quad \mathrm{G}_{\alpha \beta}=\frac{\tilde{k}}{2} \delta_{\alpha \beta}, \\
& \mathrm{H}_{\alpha \beta \gamma}=-\frac{\tilde{k}}{2} f_{\alpha \beta \gamma}, \quad \mathrm{H}_{\alpha i j}=-\frac{\tilde{k}}{2} f_{\alpha i j}, \quad \mathrm{H}_{\alpha \bar{\imath} \bar{\jmath}}=-\frac{\tilde{k}}{2} f_{\alpha \bar{\imath} \bar{\jmath}} .
\end{aligned}
$$

The $H$ gauge symmetry of the model (2.17) is manifested in the vanishing of $\bar{\alpha}$-components of the metric and the H-tensor.

As discussed in appendix B for the coset $\sigma$-model (see above eq. (B.15)), there are various approaches that can be used to treat the $H$ gauge symmetry. For example, we may take $(\hat{h}, \bar{h}) \in G \times G$ to be parametrized by the $2 \operatorname{dim} G$ - $\operatorname{dim} H$ physical degrees of freedom and understand $e^{\bar{\alpha}}=J^{\alpha}-K^{\bar{\alpha}}$ as expanded in the vielbein $\left(e^{i}, e^{\bar{\imath}}, e^{\alpha}\right)$. Alternatively, we can lift the degeneracy of the metric by setting $\mathrm{G}_{\bar{\alpha} \bar{\beta}}=\varepsilon \frac{\tilde{k}}{2} \delta_{\alpha \beta}$, then project out the $\bar{\alpha}$ directions and finally set the regulator $\varepsilon$ to zero.

\footnotetext{
${ }^{15}$ In addition to the identities in appendix A here we have $f_{\alpha \beta i}=f_{i j k}=0$ from the $\mathbb{Z}_{2}$ grading of Lie $(G)$ (see (A.4)).
} 
Using either of these methods to compute the torsionful spin connection and the corresponding curvature, the non-zero curvature components are given by

$$
\left(\tilde{\lambda}^{-2}-1\right) \hat{R}_{j k l}^{i}=-\sqrt{\tilde{\lambda}^{-2}-1} \hat{R}_{j k \bar{l}}^{i}=\hat{R}_{j \bar{k} \bar{l}}^{i}=f_{j \alpha}^{i} f_{k l}^{\alpha} .
$$

Plugging this into the RG equation (1.12), one obtains

$$
\left(\beta_{\mu \nu}^{(1)}+\beta_{\mu \nu}^{(2)}\right) d x^{\mu} \otimes d x^{\nu}=\sum_{i=\bar{\imath}=1}^{\operatorname{dim} G-\operatorname{dim} H}\left[c_{G} \tilde{\lambda}+\frac{c_{G}}{\tilde{k}} \frac{\tilde{\lambda}\left(c_{H}-\left(2 c_{G}-c_{H}\right) \tilde{\lambda}^{2}\right)}{1-\tilde{\lambda}^{2}}\right] J^{i} \otimes K^{\bar{\imath}} .
$$

Here $c_{H}$ is defined in terms of the index of the representation of $H$ in which the coset directions transform, as described in appendix A. In the case that $G$ and $H$ are simple, $c_{H}$ is proportional to the dual Coxeter number of $H$.

We conclude that only the $\tilde{\lambda}$-dependent term in (2.17) gets renormalized, i.e. the 2-loop RG equation (1.12) is solved with $X^{\mu}=Y_{\mu}=0$ and

$$
\frac{d}{d t} \tilde{k}=0, \quad \frac{d}{d t} \tilde{\lambda}=\frac{c_{G} \tilde{\lambda}}{\tilde{k}}\left[1+\frac{1}{\tilde{k}} \frac{c_{H}-\left(2 c_{G}-c_{H}\right) \tilde{\lambda}^{2}}{1-\tilde{\lambda}^{2}}\right] .
$$

The 1-loop term agrees with $[57,58]$. The level $k$ is RG-invariant, and, as expected, $\tilde{\lambda}=\infty, 0$ are fixed points. Expressing $\tilde{k}$ and $\tilde{\lambda}$ in (2.18) in terms of $k$ and the coupling $\kappa$ using (1.4) we find

$$
\frac{d}{d t} k=0, \quad \frac{d}{d t} \kappa=\frac{c_{G}}{2 k}\left[\left(1-\kappa^{2}\right)+\frac{c_{G}(1-\kappa)^{2}\left(1+4 \kappa-\kappa^{2}\right)-c_{H}\left(1-\kappa^{4}\right)}{2 k \kappa}\right] .
$$

The fixed point $\kappa=1$ corresponds to $\lambda=0(\tilde{\lambda}=\infty)$, that is when the $\lambda$-model reduces to the $G / H$ gWZW model. The other fixed point, which corresponds to the $G / H$ gWZW model with level $-k$, is corrected and is given by $\kappa=-\left(1+\frac{4 c_{G}}{k}\right)$ (cf. (2.18)).

As in the group space case (2.27) the 2-loop term in the $\beta$-function (2.37) is, in general, scheme-dependent. Again, we find that the symmetry under $k \rightarrow-k, \lambda \rightarrow \lambda^{-1}$ in (1.7), present at the 1-loop order is not there in the 2-loop term of (2.37). However, after introducing the shifted level $\mathrm{k}=\tilde{k}-c_{G}=k+c_{G}$ as in (2.28), the 2-loop RG equation for $\tilde{\lambda}$ (2.37) becomes

$$
\frac{d}{d t} \tilde{\lambda}=\frac{c_{G} \tilde{\lambda}}{\mathrm{k}}\left[1-\frac{1}{\mathrm{k}}\left(c_{G}-c_{H}\right) \frac{1+\tilde{\lambda}^{2}}{1-\tilde{\lambda}^{2}}\right],
$$

which is manifestly invariant under $\mathrm{k} \rightarrow-\mathrm{k}, \tilde{\lambda} \rightarrow \tilde{\lambda}^{-1}$, a quantum version of the symmetry (1.7) of the original couplings (cf. (2.30)).

In the NAD limit (1.9) we get from (2.38)

$$
\frac{d}{d t} \mathrm{~h}=2 c_{G}+4 c_{G}\left(c_{G}-c_{H}\right) \mathrm{h}^{-1}
$$

with the 2-loop coefficient being scheme-independent in this 1-coupling limit as in the group space case (2.31). As verified in appendix B, (2.40) matches the expression for the 2-loop $\beta$-function of the $G / H$ symmetric space $\sigma$-model (reproducing in particular cases the results in $[62,65-67])$. This demonstrates that the symmetric space $\sigma$-model and its non-abelian dual have the same 2-loop $\beta$-function. 


\subsection{Low-dimensional examples: $\lambda$-models for $\mathrm{SU}(2)$ and $\mathrm{SU}(2) / \mathrm{U}(1)$}

Let us first study the simplest case of the model (2.10), i.e. with $G=\mathrm{SU}(2)$. Parametrizing

$$
\hat{h}=\exp \left(i \phi \sigma_{3}\right) \exp \left(i \theta \sigma_{1}\right) \exp \left(i \psi \sigma_{3}\right), \quad \bar{h}=\exp \left(i \bar{\phi} \sigma_{3}\right) \exp \left(i \bar{\theta} \sigma_{1}\right) \exp \left(i \bar{\psi} \sigma_{3}\right),
$$

where $\sigma_{a}$ are the standard Pauli matrices, we find the following 6-dimensional metric and B-field $\left(\mathrm{G} \equiv \mathrm{G}_{\mu \nu} d x^{\mu} d x^{\nu}, \mathrm{B} \equiv \frac{1}{2} \mathrm{~B}_{\mu \nu} d x^{\mu} \wedge d x^{\nu}\right)$

$$
\begin{aligned}
\mathrm{G}_{=}= & \mathrm{G}_{0}+\mathrm{G}_{1}, \quad \mathrm{~B}=\mathrm{B}_{0}+\mathrm{B}_{1}, \\
\mathrm{G}_{0}= & -\tilde{k}\left(d \theta^{2}+d \phi^{2}+d \psi^{2}+2 \cos 2 \theta d \phi d \psi+d \bar{\theta}^{2}+d \bar{\phi}^{2}+d \bar{\psi}^{2}+2 \cos 2 \bar{\theta} d \bar{\phi} d \bar{\psi}\right), \\
\mathrm{B}_{0}= & -\tilde{k}(\cos 2 \theta d \phi \wedge d \psi+\cos 2 \bar{\theta} d \bar{\phi} \wedge d \bar{\psi}), \\
\mathrm{G}_{1}= & -2 \tilde{k} \tilde{\lambda}[\cos 2(\psi+\bar{\phi})(d \theta d \bar{\theta}-\sin 2 \theta \sin 2 \bar{\theta} d \phi d \bar{\psi})+(d \psi+\cos 2 \theta d \phi)(d \bar{\phi}+\cos 2 \bar{\theta} d \bar{\psi}) \\
& +\sin 2(\psi+\bar{\phi})(\sin 2 \theta d \phi d \bar{\theta}+\sin 2 \bar{\theta} d \theta d \bar{\psi})] \\
\mathrm{B}_{1}= & -\tilde{k} \tilde{\lambda}[\cos 2(\psi+\bar{\phi})(d \theta \wedge d \bar{\theta}-\sin 2 \theta \sin 2 \bar{\theta} d \phi \wedge d \bar{\psi})+(d \psi+\cos 2 \theta d \phi) \wedge(d \bar{\phi}+\cos 2 \bar{\theta} d \bar{\psi}) \\
& +\sin 2(\psi+\bar{\phi})(\sin 2 \theta d \phi \wedge d \bar{\theta}+\sin 2 \bar{\theta} d \theta \wedge d \bar{\psi})] .
\end{aligned}
$$

One can check explicitly that this metric and B-field solve the 2-loop RG equation (1.12) with vanishing $X^{\mu}$ and $Y_{\mu}$, and the couplings running as in (2.26) (with the dual Coxeter number of $G=\mathrm{SU}(2)$ given by $\left.c_{G}=2\right)$.

Next, let us consider the $\lambda$-model (2.17) for the symmetric space $G / H=\mathrm{SU}(2) / \mathrm{U}(1)$. Using the parametrization for $\hat{h}$ and $\bar{h}$ given in eq. (2.41), we find that the corresponding target space background depends on $\psi$ and $\bar{\phi}$ only through $\chi=\psi+\bar{\phi}$, which is a manifestation of the U(1) gauge symmetry. The resulting 5-d metric and B-field are

$$
\begin{aligned}
\mathrm{G}= & \mathrm{G}_{0}+\mathrm{G}_{1}, \quad \mathrm{~B}=\mathrm{B}_{0}+\mathrm{B}_{1}, \\
\mathrm{G}_{0}= & -\tilde{k}\left(d \theta^{2}+d \phi^{2}+d \bar{\theta}^{2}+d \bar{\psi}^{2}\right), \quad \mathrm{B}_{0}=0, \\
\mathrm{G}_{1}= & -2 \tilde{k} \tilde{\lambda}[\cos 2 \chi(d \theta d \bar{\theta}-\sin 2 \theta \sin 2 \bar{\theta} d \phi d \bar{\psi})+\sin 2 \chi(\sin 2 \theta d \phi d \bar{\theta}+\sin 2 \bar{\theta} d \theta d \bar{\psi})] \\
& +\tilde{k} d \chi^{2}-2 \tilde{k}(d \chi+\cos 2 \theta d \phi)(d \chi+\cos 2 \bar{\theta} d \bar{\psi}), \\
\mathrm{B}_{1}= & -\tilde{k} \tilde{\lambda}[\cos 2 \chi(d \theta \wedge d \bar{\theta}-\sin 2 \theta \sin 2 \bar{\theta} d \phi \wedge d \bar{\psi})+\sin 2 \chi(\sin 2 \theta d \phi \wedge d \bar{\theta}+\sin 2 \bar{\theta} d \theta \wedge d \bar{\psi})] \\
& -\tilde{k}(d \chi+\cos 2 \theta d \phi) \wedge(d \chi+\cos 2 \bar{\theta} d \bar{\psi}) .
\end{aligned}
$$

Again one can check explicitly that this metric and B-field solve the 2-loop RG equation (1.12) with vanishing $X^{\mu}$ and $Y_{\mu}$, and the couplings running as in (2.37) (with the dual Coxeter number for $G=\mathrm{SU}(2)$ given by $c_{G}=2$ and $c_{H}=0$ for $\left.H=\mathrm{U}(1)\right)$.

\section{Renormalization of $\lambda$-model: standard configuration space}

In section 2 we demonstrated the 2-loop renormalizability of the $\lambda$-model (1.2) for general groups $G$ and symmetric spaces $G / H$. It is then natural to ask what this implies for the model on the standard or physical configuration space, i.e. the $\sigma$-model found by integrating out $A_{ \pm}$in (1.2). Doing so classically gives the following Lagrangian

$$
\mathcal{L}=k\left(\mathcal{L}_{\mathrm{PCM}}(g)+\mathcal{L}_{\mathrm{WZ}}(g)+\operatorname{Tr}\left[J_{+} M^{-1} K_{-}\right]\right), \quad M=\operatorname{Ad}_{g}-I+\left(1-\lambda^{-1}\right) P .
$$


Similarly, for the NAD model (1.10) we find

$$
\mathcal{L}=-\frac{1}{2} \mathrm{~h} \operatorname{Tr}\left[\partial_{+} v \mathcal{M}^{-1} \partial_{-} v\right], \quad \mathcal{M}=\operatorname{ad}_{v}+P .
$$

The integration over $A_{ \pm}$may also give rise to quantum counterterms required to preserve the renormalizability of (3.1) at 2 loops [7]. It is natural to expect that, since the term quadratic in $A_{ \pm}$in the Lagrangian (1.2) has the form $\operatorname{Tr}\left[A_{+} M A_{-}\right]$, these corrections may depend on the matrix $M$ in (3.1), but determining their form in general appears to be nontrivial. Here we will focus on the examples of the $\lambda$-model for the $\mathrm{SU}(2) / \mathrm{U}(1)$ symmetric space and $\mathrm{SU}(2)$ group space.

\section{$3.1 \mathrm{SU}(2) / \mathrm{U}(1)$}

The $\lambda$-model for $\mathrm{SU}(2) / \mathrm{U}(1)$ is related by analytic continuation to that of $\mathrm{SU}(1,1) / \mathrm{U}(1)$, which was studied in detail in [7]. Here we briefly summarize certain key points of the discussion there. Fixing the U(1) gauge symmetry by choosing the following parametrization of the coset element

$$
g=\exp \left(i \alpha \sigma_{3}\right) \exp \left(i \beta \sigma_{2}\right), \quad \cos \alpha=\sqrt{p^{2}+q^{2}}, \quad \tan \beta=\frac{p}{q},
$$

the $\sigma$-model (3.1) yields the following classical metric (the B-field is trivial in $2 \mathrm{~d}$ target space and $\kappa$ is defined in (1.4))

$$
\mathrm{G}_{0}=\frac{k}{1-p^{2}-q^{2}}\left(\kappa d p^{2}+\kappa^{-1} d q^{2}\right) .
$$

The observation in [7] was that this metric should be modified by a particular quantum correction from the determinant [68] resulting from integrating over $A_{ \pm}$

$$
\delta \mathrm{G}=-\frac{1}{2}(d \log \operatorname{det} M)^{2}=-\frac{1}{2}\left[d \log \left(1-p^{2}-q^{2}\right)\right]^{2} .
$$

The 1-loop corrected background $\mathrm{G}=\mathrm{G}_{0}+\delta \mathrm{G}$ then solves the 2-loop RG equation (1.12) with

$$
\frac{d}{d t} k=0+\frac{1}{k} \frac{\left(1-\kappa^{2}\right)^{2}}{\kappa^{2}}, \quad \frac{d}{d t} \kappa=\frac{1}{k}\left(1-\kappa^{2}\right)
$$

and

$$
X^{p}=-\frac{p}{k \kappa}\left[1-\frac{\kappa}{k}+\frac{\kappa^{-1} p^{2}+\kappa q^{2}}{k\left(1-p^{2}-q^{2}\right)}\right], \quad X^{q}=-\frac{q \kappa}{k}\left[1-\frac{1}{k \kappa}+\frac{\kappa^{-1} p^{2}+\kappa q^{2}}{k\left(1-p^{2}-q^{2}\right)}\right], \quad Y_{p, q}=0 .
$$

In this analysis the symmetry (1.7) of the 1-loop RG equation in (3.6) survives at the 2-loop level. Indeed, while the leading 1-loop terms in (3.6) agree with the RG equations (2.38) found from the analysis on the extended configuration space, they deviate at the 2-loop order. Since the 2-loop terms in a two-coupling theory are generally scheme-dependent, we 
can, in fact, match the two $\beta$-function expressions by redefining the parameters in (3.4) as follows ${ }^{16}$

$$
k \rightarrow k-\frac{(1-\kappa)^{2}}{\kappa}, \quad \kappa \rightarrow \kappa+\frac{4(1-\kappa)}{k} .
$$

Note that in the $\kappa \rightarrow 1$ limit the level $k$ remains unmodified, in agreement with this limit corresponding to the $\mathrm{SU}(2) / \mathrm{U}(1)$ gWZW model.

\section{$3.2 \mathrm{SU}(2)$}

Let us now return to the $\lambda$-model for the group $\mathrm{SU}(2)$. Parametrizing the group element as

$$
g=\exp \left[-i \arcsin \alpha\left(\cos \beta \sigma_{2}+\sin \beta\left(\cos \gamma \sigma_{3}-\sin \gamma \sigma_{1}\right)\right)\right],
$$

we obtain from (3.1) the following $3 \mathrm{~d}$ classical $\sigma$-model metric and B-field $[8]^{17}$

$$
\begin{aligned}
& \mathrm{G}_{0}=k\left[\frac{d \alpha^{2}}{\kappa\left(1-\alpha^{2}\right)}+\frac{\kappa \alpha^{2}}{\Delta}\left(d \beta^{2}+\sin ^{2} \beta d \gamma^{2}\right)\right], \quad \Delta \equiv \kappa^{2}+\left(1-\kappa^{2}\right) \alpha^{2}, \\
& \mathrm{~B}_{0}=k\left(\arcsin \alpha-\frac{\kappa^{2} \alpha \sqrt{1-\alpha^{2}}}{\Delta}\right) \sin \beta d \beta \wedge d \gamma, \\
& \mathrm{H}_{0}=d \mathrm{~B}_{0}=\frac{k \alpha^{2}}{\sqrt{1-\alpha^{2}} \Delta^{2}}\left[2 \kappa^{2}+\left(1-\kappa^{2}\right) \Delta\right] \sin \beta d \alpha \wedge d \beta \wedge d \gamma
\end{aligned}
$$

It is possible to ensure the 2-loop renormalizability of the model by adding to this classical background special quantum counterterms. The resulting 1-loop $(1 / k)$ corrected background is

$$
\begin{aligned}
& \mathrm{G}=\mathrm{G}_{0}+\frac{2\left(1-\kappa^{2}\right)^{2} \alpha^{4}}{\kappa^{2}\left(1-\alpha^{2}\right) \Delta^{2}} d \alpha^{2}, \\
& \mathrm{~B}=\frac{\mathrm{k}}{k} \mathrm{~B}_{0}-2\left(\arctan \frac{\alpha}{\kappa \sqrt{1-\alpha^{2}}}-\frac{\kappa \alpha \sqrt{1-\alpha^{2}}}{\Delta}\right) \sin \beta d \beta \wedge d \gamma, \\
& \mathrm{H}=\frac{\mathrm{k}}{k} \mathrm{H}_{0}-\frac{4 \kappa \alpha^{2}}{\sqrt{1-\alpha^{2} \Delta^{2}}} \sin \beta d \alpha \wedge d \beta \wedge d \gamma, \quad \mathrm{k} \equiv k+\frac{4+\left(1+\kappa^{2}\right)^{2}}{4 \kappa} .
\end{aligned}
$$

This corrected background (3.11) solves the 2-loop RG equation (1.12) with

$$
\frac{d}{d t} k=0+\frac{\left(1-\kappa^{2}\right)^{3}\left(5+3 \kappa^{2}\right)}{8 k \kappa^{2}}, \quad \frac{d}{d t} \kappa=\frac{\left(1-\kappa^{2}\right)^{2}}{2 k}\left[1-\frac{\left(1-\kappa^{2}\right)^{2}}{k \kappa}\right],
$$

and the only non-zero component of $X^{\mu}$ being $\left(Y_{\mu}=0\right)$

$$
X^{\alpha}=\frac{\alpha\left(1-\alpha^{2}\right)\left(1-\kappa^{2}\right)}{k \Delta}\left[\kappa+\frac{2 \alpha^{2} \kappa^{2}\left(1-\kappa^{2}\right)-\left(3-2 \kappa^{2}+\kappa^{4}\right) \Delta^{2}}{k \Delta^{2}}\right] .
$$

\footnotetext{
${ }^{16}$ The most general redefinition achieving this is $\left(C_{1}\right.$ and $C_{2}$ are free constants $) k \rightarrow k-\frac{(1-\kappa)^{2}}{\kappa}+2 C_{1}$ and $\kappa \rightarrow \kappa+\frac{4}{k}\left[(1-\kappa)\left(1-(1+\kappa) C_{2}\right)-2 C_{1}\left(1-\kappa^{2}\right) \operatorname{arctanh} \kappa\right]$.

${ }^{17}$ The WZ term in $k \mathcal{L}_{\mathrm{WZ}}(g)$ contributes $k\left(\arcsin \alpha-\alpha \sqrt{1-\alpha^{2}}\right)$ to the B-field.
} 
Again, in this analysis the symmetry (1.7) of the 1-loop RG equation in (3.6) survives at the 2-loop level and, while the 1-loop $\beta$-functions for $k$ and $\kappa$ match those found in the extended configuration space approach, i.e. (2.27) with $c_{G}=2$, the matching of the 2-loop terms is only achieved after the following redefinition ${ }^{18}$

$$
k \rightarrow k-\frac{4+\left(1+\kappa^{2}\right)^{2}}{4 \kappa}+2, \quad \kappa \rightarrow \kappa-\frac{(1-\kappa)^{2}(1+\kappa)}{k} .
$$

As in (3.8), the level $k$ is not modified in the WZW limit $\kappa \rightarrow 1$.

Note that the coupling $\mathrm{k}$ defined in (3.11) does not run at 2-loop order, $\frac{d}{d t} \mathrm{k}=0$. This is consistent with the fact that $k$ does not run in the extended configuration space approach and the particular shift (3.14) required to recover (2.27) from (3.12). This RG invariant $\mathrm{k}$ is the coefficient of $\arcsin \alpha$ (present in $\mathrm{B}_{0}$ in (3.10)) in the 1-loop corrected background (3.11). Choosing it to be integer-valued removes the global ambiguities arising from the $\arcsin \alpha$ term. Furthermore, given that under a large transformation ${ }^{19}$

$$
\delta(\arcsin \alpha)=\operatorname{sign} \kappa \delta\left(\arctan \frac{\alpha}{\kappa \sqrt{1-\alpha^{2}}}\right),
$$

and that the coefficient of $\arctan \frac{\alpha}{\kappa \sqrt{1-\alpha^{2}}}$ in (3.11) is integer-valued, this term does not lead to any additional ambiguities. The quantization of the flux

$$
\frac{1}{4 \pi^{2}} \int \mathrm{H}_{0}=k, \quad \frac{1}{4 \pi^{2}} \int \mathrm{H}=\mathrm{k}-2,
$$

also supports the identification of $\mathrm{k}$ as integer-valued.

In the case of the $\mathrm{SU}(2) / \mathrm{U}(1) \lambda$-model it was possible to write the 1-loop corrections in a simple way (3.5) in terms of the matrix $M$ as defined in (3.1). Let us try to do the same for the 1-loop corrections (3.11) to the $\mathrm{SU}(2) \lambda$-model. Since the $\mathrm{SU}(2) / \mathrm{U}(1)$ counterterm (3.5) took the form $\Delta \mathcal{L}=\frac{1}{2}(\partial \log \operatorname{det} M)^{2}=\frac{1}{2}\left(\operatorname{Tr}\left[M^{-1} \partial M\right]\right)^{2}$, we may consider counterterms (with coefficients $c_{i}$ ) built out of the quantity $M^{-1} \partial M$. In addition, we may also include counterterms (with coefficients $d_{i}$ ) proportional to the terms present in the classical Lagrangian (3.1) (and its image under parity). As a result, we are led to the following ansatz

$$
\begin{aligned}
\Delta \mathcal{L}= & c_{1}\left(\partial_{+} \log \operatorname{det} M\right)\left(\partial_{-} \log \operatorname{det} M\right)+c_{2} \mathcal{L}_{\mathrm{PCM}}(M)+c_{3} \mathcal{L}_{\mathrm{WZ}}(M) \\
& +d_{1} \mathcal{L}_{\mathrm{PCM}}(g)+d_{2} \mathcal{L}_{\mathrm{WZ}}(g)+d_{3} \operatorname{Tr}\left[J_{+} M^{-1} K_{-}\right]+d_{4} \operatorname{Tr}\left[J_{-} M^{-1} K_{+}\right]
\end{aligned}
$$

We find that this matches the required 1-loop corrected background (3.11) provided the constants $c_{i}$ and $d_{i}$ take the following values

$$
\begin{aligned}
& c_{1}=-\frac{3+\kappa}{2(1-\kappa)}, \quad c_{2}=-\frac{2(1+\kappa)}{1-\kappa}, \quad c_{3}=1, \\
& d_{1}=0, \quad d_{2}=\mathrm{k}-k-2, \quad d_{3}=\frac{1}{2}\left[\mathrm{k}-k+\left(1+\kappa^{-1}\right)\right], \quad d_{4}=-\frac{1}{2}\left[\mathrm{k}-k-3\left(1+\kappa^{-1}\right)\right] .
\end{aligned}
$$

\footnotetext{
${ }^{18}$ The most general redefinition achieving this is $\left(C_{1}\right.$ and $C_{2}$ are free constants $) k \rightarrow k-\frac{4+\left(1+\kappa^{2}\right)^{2}}{4 \kappa}+2 C_{1}$ and $\quad \kappa \rightarrow \kappa-\frac{1}{k}\left[(1-\kappa)^{2}(1+\kappa)\left(1+(1+\kappa) C_{2}\right)+\left(C_{1}-1\right)\left(1-\kappa^{2}\right)\left(\left(1-\kappa^{2}\right) \operatorname{arctanh} \kappa+\kappa\right)\right]$.

${ }^{19}$ Note that $\arctan \frac{\alpha}{\kappa \sqrt{1-\alpha^{2}}}=\operatorname{sign} \kappa \arcsin \frac{\alpha}{\sqrt{\Delta}}$ and $\alpha=\frac{\alpha}{\sqrt{\Delta}}$ for $\alpha=0, \pm 1$.
} 
Combining the quantum counterterms (3.17) with the classical Lagrangian (3.1) allows us to represent the $\sigma$-model corresponding to the 1-loop corrected geometry (3.11) in the form

$$
\begin{aligned}
\mathcal{L}=k & \mathcal{L}_{\mathrm{PCM}}(g)+(\mathrm{k}-2) \mathcal{L}_{\mathrm{WZ}}(g) \\
& +\frac{1}{2} \operatorname{Tr}\left[\left(\mathrm{k}+k+\left(1+\kappa^{-1}\right)\right) J_{+} M^{-1} K_{-}-\left(\mathrm{k}-k-3\left(1+\kappa^{-1}\right)\right) J_{-} M^{-1} K_{+}\right] \\
& -\frac{3+\kappa}{2(1-\kappa)}\left(\partial_{+} \log \operatorname{det} M\right)\left(\partial_{-} \log \operatorname{det} M\right)-\frac{2(1+\kappa)}{1-\kappa} \mathcal{L}_{\mathrm{PCM}}(M)+\mathcal{L}_{\mathrm{WZ}}(M) .
\end{aligned}
$$

Note that in the WZW limit $\kappa \rightarrow 1$ when the RG invariant $\mathrm{k}$ in (3.11) reduces to the usual shift of the level

$$
\left.\mathrm{k}\right|_{\kappa=1}=k+2
$$

the other corrections to the metric and B-field in (3.11) vanish, so that the expression in (3.19) indeed reduces to the standard WZW Lagrangian $k\left[\mathcal{L}_{\mathrm{PCM}}(g)+\mathcal{L}_{\mathrm{WZ}}(g)\right] .{ }^{20}$

\subsection{1 $\mathrm{SU}(2) \times \mathrm{U}(1)$ invariant limit: abelian T-duality to squashed 3-sphere}

Let us consider the formal limit

$$
\alpha \rightarrow \sin (\alpha+i \zeta), \quad \zeta \rightarrow \infty
$$

in which the 1-loop corrected background (3.11) becomes $^{21}$

$$
\begin{aligned}
\mathrm{G} & =\left(\frac{k}{\kappa}+\frac{2}{\kappa^{2}}\right) d \alpha^{2}+\frac{k \kappa}{1-\kappa^{2}}\left(d \beta^{2}+\sin ^{2} \beta d \gamma^{2}\right) \\
\mathrm{B} & =\left[k+\frac{4+\left(1+\kappa^{2}\right)^{2}}{4 \kappa}\right] \alpha \sin \beta d \beta \wedge d \gamma, \quad \mathrm{H}=\left[k+\frac{4+\left(1+\kappa^{2}\right)^{2}}{4 \kappa}\right] \sin \beta d \alpha \wedge d \beta \wedge d \gamma .
\end{aligned}
$$

The resulting metric and $\mathrm{H}$-tensor have $\mathrm{SU}(2) \times \mathrm{U}(1)$ symmetry (while, as usual, the B-field cannot be written in a way that is manifestly invariant). The background (3.22) solves the 2-loop RG flow equations (1.12) with the parameters running as in (3.12) and $X^{\mu}=Y_{\mu}=0$.

Focusing on the classical part of (3.22) by taking $k$ large, we may shift the B-field by a closed 2-form in order to make translations in $\alpha$ a manifest symmetry

$$
\mathrm{G}=k\left[\frac{1}{\kappa} d \alpha^{2}+\frac{\kappa}{1-\kappa^{2}}\left(d \beta^{2}+\sin ^{2} \beta d \gamma^{2}\right)\right], \quad \mathrm{B}=k \cos \beta d \alpha \wedge d \gamma .
$$

Applying T-duality in the $\alpha$ direction we find the metric of the squashed 3-sphere (which also has the interpretation of the $\eta$-deformation of the SU(2) PCM, cf. [69])

$$
\tilde{\mathrm{G}}=k\left[\kappa(d \tilde{\alpha}-\cos \beta d \gamma)^{2}+\frac{\kappa}{1-\kappa^{2}}\left(d \beta^{2}+\sin ^{2} \beta d \gamma^{2}\right)\right] .
$$

\footnotetext{
${ }^{20}$ While the coefficients $\frac{3+\kappa}{2(1-\kappa)}$ and $\frac{2(1+\kappa)}{1-\kappa}$ blow up in the limit $\kappa \rightarrow 1$, the corresponding $M$-dependent expressions vanish faster. Note also that $\arcsin \alpha=\arctan \frac{\alpha}{\sqrt{1-\alpha^{2}}}$.

${ }^{21}$ We have dropped trivial (i.e. closed 2-form) contributions to the B-field such that only the $\arcsin \alpha \rightarrow$ $\alpha+i \zeta \sim \alpha$ term in (3.11) gives a relevant contribution in this limit.
} 
To extend this relation to the quantum level, let us use the 1-loop corrected form of the T-duality transformation given in [70] (see also [71, 72]) in an alternative scheme related to ours by

$$
(\mathrm{G}+\mathrm{B})_{\mu \nu} \rightarrow(\mathrm{G}+\mathrm{B})_{\mu \nu}+\frac{1}{4} \mathrm{H}_{\mu \nu}^{2} .
$$

Starting with the background (3.22) and implementing this scheme change gives

$$
\begin{aligned}
& \mathrm{G}=\left(\frac{k}{\kappa}+\frac{2}{\kappa^{2}}+\frac{\left(1-\kappa^{2}\right)^{2}}{2 \kappa^{2}}\right) d \alpha^{2}+\left(\frac{k \kappa}{1-\kappa^{2}}+\frac{1-\kappa^{2}}{2}\right)\left(d \beta^{2}+\sin ^{2} \beta d \gamma^{2}\right), \\
& \mathrm{B}=\left(k+\frac{4+\left(1+\kappa^{2}\right)^{2}}{4 \kappa}\right) \cos \beta d \alpha \wedge d \gamma .
\end{aligned}
$$

This is a special case of a general ansatz

$$
\begin{aligned}
\mathrm{G} & =e^{\varphi}\left(d \alpha+V_{m} d x^{m}\right)^{2}+\mathrm{G}_{m n}^{\prime} d x^{m} d x^{n}, & \mathrm{G}_{m n}^{\prime} & =\mathrm{G}_{m n}-e^{\varphi} V_{m} V_{n}, \\
\mathrm{~B} & =W_{m} d x^{m} \wedge d \alpha+\frac{1}{2} \mathrm{~B}_{m n} d x^{m} \wedge d x^{n}, & x^{m} & =\{\beta, \gamma\},
\end{aligned}
$$

where $\varphi$ is a constant, $V_{m}=0$ and $\mathrm{B}_{m n}=0$. Then the 1-loop corrected T-duality transformation rules simplify to $\left(W_{m n} \equiv \partial_{m} W_{n}-\partial_{n} W_{m}\right)$

$\tilde{\varphi}=-\varphi+\frac{1}{4} e^{-\varphi} W_{m n} W^{m n}, \quad \tilde{V}_{m}=W_{m}, \quad \tilde{\mathrm{G}}_{m n}^{\prime}=\mathrm{G}_{m n}^{\prime}, \quad \tilde{W}_{m}=0, \quad \tilde{\mathrm{B}}_{m n}=0$,

where the $\frac{1}{4} e^{-\varphi} W_{m n} W^{m n}$ term is the 1-loop (or $\alpha^{\prime} \sim \frac{1}{k}$ ) correction [70]. As a result, the T-dual background is found to be

$$
\begin{aligned}
& \tilde{\mathrm{G}}=\left[k \kappa+\frac{1}{2}\left(1+\kappa^{2}\right)^{2}\right](d \tilde{\alpha}-\cos \beta d \gamma)^{2}+\left[\frac{k \kappa}{1-\kappa^{2}}+\frac{1}{2}\left(1-\kappa^{2}\right)\right]\left(d \beta^{2}+\sin ^{2} \beta d \gamma^{2}\right), \\
& \tilde{\mathrm{B}}=0,
\end{aligned}
$$

where we have rescaled $\tilde{\alpha} .^{22}$ This background (3.29) indeed solves the 2-loop RG equations (1.12) with the parameters running as in (3.12) and $X^{\tilde{\alpha}, \beta, \gamma}=Y_{\tilde{\alpha}, \beta, \gamma}=0$.

On symmetry grounds, the $\sigma$-model for the squashed 3 -sphere (3.24) is renormalizable to all loop orders (without the need for counterterms of different form) [20] (see also appendix B). Indeed, this is consistent with (3.29) as the coupling redefinition

$$
k=\hat{k}-\frac{3+\hat{\kappa}^{4}}{2 \hat{\kappa}}, \quad \kappa=\hat{\kappa}+\frac{1-\hat{\kappa}^{2}}{\hat{k}},
$$

gives simply (cf. (3.24))

$$
\tilde{\mathrm{G}}=\hat{k}\left[\hat{\kappa}(d \tilde{\alpha}-\cos \beta d \gamma)^{2}+\frac{\hat{\kappa}}{1-\hat{\kappa}^{2}}\left(d \beta^{2}+\sin ^{2} \beta d \gamma^{2}\right)\right] .
$$

We thus find another example (in addition to the one discussed in [7]) of how the required loop corrections to the T-duality transformation rules naturally appear from the deformation under the RG flow of more general integrable models. Similar higher-loop corrections are expected for non-abelian (and also Poisson-Lie) duality.

\footnotetext{
${ }^{22}$ As the dual B-field is vanishing, undoing the scheme change (3.25) has no effect.
} 


\subsubsection{Non-abelian dual of SU(2) PCM}

In the case of the $\mathrm{SU}(2) \lambda$-model in the coordinates (3.10), the NAD limit (1.9) amounts to

$$
\alpha \rightarrow \kappa \alpha, \quad \kappa \rightarrow \frac{1}{4} \mathrm{~h} k^{-1}, \quad k \rightarrow \infty,
$$

where $\mathrm{h}$ and the new coordinate $\alpha$ are fixed. In this limit the 1-loop-corrected background (3.11) becomes

$$
\begin{aligned}
\mathrm{G} & =\left[\frac{\mathrm{h}}{4}+\frac{2 \alpha^{4}}{\left(1+\alpha^{2}\right)^{2}}\right] d \alpha^{2}+\frac{h}{4} \frac{\alpha^{2}}{\left(1+\alpha^{2}\right)}\left(d \beta^{2}+\sin ^{2} \beta d \gamma^{2}\right), \\
\mathrm{B} & =\left[\frac{\mathrm{h}+5}{4} \frac{\alpha^{3}}{\left(1+\alpha^{2}\right)}+\frac{2 \alpha}{1+\alpha^{2}}-2 \arctan \alpha\right] \sin \beta d \beta \wedge d \gamma, \\
\mathrm{H} & =\left[\frac{\mathrm{h}+5}{4} \frac{\alpha^{2}\left(3+\alpha^{2}\right)}{4\left(1+\alpha^{2}\right)^{2}}-\frac{4 \alpha^{2}}{\left(1+\alpha^{2}\right)^{2}}\right] \sin \beta d \alpha \wedge d \beta \wedge d \gamma .
\end{aligned}
$$

It solves the 2-loop RG equations (1.12) with

$$
\begin{gathered}
\frac{d}{d t} \mathrm{~h}=2+2 \mathrm{~h}^{-1}, \\
X^{\alpha}=\frac{2 \alpha}{\mathrm{h}\left(1+\alpha^{2}\right)}\left[\left(1-\alpha^{2}\right)-\frac{4\left(5+5 \alpha^{2}+3 \alpha^{4}+\alpha^{6}\right)}{h\left(1+\alpha^{2}\right)^{2}}\right], \quad X^{\beta, \gamma}=Y_{\alpha, \beta, \gamma}=0 .
\end{gathered}
$$

The RG equation (3.34) for $\mathrm{h}$ follows also by taking the limit (3.32) in (3.12). It matches the 2-loop running of the coupling in the SU(2) PCM (cf. (B.11)). The limit of the diffeomorphism vector $X^{\alpha}(3.13)$ gives (3.35) after taking into account an extra contribution due to the RG-dependent rescaling of $\alpha$ in (3.32).

We can also take the NAD limit (1.9) in the corrected Lagrangian (3.19), thus getting

$$
\begin{aligned}
\mathcal{L}=- & \left(\frac{1}{2} \mathrm{~h}+\frac{9}{4}\right) \operatorname{Tr}\left[\partial_{+} v \mathcal{M}^{-1} \partial_{-} v\right]-\frac{7}{4} \operatorname{Tr}\left[\partial_{-} v \mathcal{M}^{-1} \partial_{+} v\right] \\
& -\frac{3}{2}\left(\partial_{+} \log \operatorname{det} \mathcal{M}\right)\left(\partial_{-} \log \operatorname{det} \mathcal{M}\right)-2 \operatorname{PCM}(\mathcal{M})+\operatorname{WZ}(\mathcal{M}) .
\end{aligned}
$$

Setting

$$
v=-\frac{i}{2} \alpha\left[\cos \beta \sigma_{2}+\sin \beta\left(\cos \gamma \sigma_{3}-\sin \gamma \sigma_{1}\right)\right],
$$

we recover the expected $\sigma$-model with couplings given by (3.33).

Taking the further limit of infinite shift of $\alpha$, i.e.

$$
\alpha \rightarrow \alpha+\ell, \quad \ell \rightarrow \infty
$$

in (3.33), we find

$$
\mathrm{G}=\frac{\mathrm{h}+8}{4} d \alpha^{2}+\frac{\mathrm{h}}{4}\left(d \beta^{2}+\sin ^{2} \beta d \gamma^{2}\right), \quad \mathrm{B}=\frac{\mathrm{h}+5}{4} \cos \beta d \alpha \wedge d \gamma,
$$

where we have dropped a trivial (closed 2-form) contribution to the B-field in order to make the shift-symmetry of $\alpha$ manifest. The resulting background is thus $\mathbb{R} \times S^{2}$ supported by 
a constant H-tensor. Changing scheme as in (3.25), T-dualising in $\alpha$ using the rules in eq. (3.28) and rescaling $\tilde{\alpha}$ we find ${ }^{23}$

$$
\begin{aligned}
\tilde{\mathrm{G}} & =\frac{\mathrm{h}+2}{4}\left(d \beta^{2}+d \tilde{\alpha}^{2}+d \gamma^{2}-2 \cos \beta d \tilde{\alpha} d \gamma\right) \\
& =(\mathrm{h}+2)\left(d \theta^{2}+\sin ^{2} \theta d \psi^{2}+\cos ^{2} \theta d \chi^{2}\right),
\end{aligned}
$$

i.e. the $\mathrm{SU}(2) \mathrm{PCM}$ with the $S^{3}$ radius-squared equal to $\mathrm{h}+2 .{ }^{24}$ Here $\tilde{\alpha}=\psi+\chi, \gamma=$ $\psi-\chi$ and $\beta=2 \theta$. Let us also note that in the limit $\alpha \rightarrow \varepsilon \alpha, \mathrm{h} \rightarrow \varepsilon^{-2} \mathrm{~h}, \varepsilon \rightarrow 0$, the background (3.33) becomes flat with vanishing $H$.

\section{Concluding remarks}

As we have seen above, formulating the $\lambda$-model on extended $(G \times G \times G)$ configuration space "linearizes" the RG flow, i.e. makes its renormalizability manifest without the need for extra local counterterms apart from running of the coupling $\lambda$. The same is true in the limit (1.9) that gives the interpolating model for non-abelian duality. ${ }^{25}$ Using this relation we demonstrated that the PCM and symmetric space $\sigma$-model have the same 2-loop $\beta$ functions as their non-abelian duals, thereby extending their quantum equivalence to the 2-loop level.

One open problem is how to interpret the local counterterms required for 2-loop renormalizability of the $\lambda$-model defined on the standard configuration space (3.1) starting from the manifestly renormalizable theory on the extended configuration space (2.2). In the simplest example of $\mathrm{SU}(2) / \mathrm{U}(1)$ model the origin of the counterterm (3.5) can be traced to the determinant resulting from integrating out the $2 \mathrm{~d}$ gauge field [7]. However, in the $\mathrm{SU}(2)$ model with $3 \mathrm{~d}$ target space the derivation and structure of the rather intricate counterterms in (3.19) and (3.36) are not immediately clear.

Another interesting question is to understand how integrability implies renormalizability and if renormalizable $\sigma$-models should always be integrable. ${ }^{26}$ Whether this relationship should be with classical or quantum integrability is also of interest. Indeed, there are

\footnotetext{
${ }^{23}$ Note that, since we now have a 1-coupling theory, the 2-loop $\beta$-function (3.34) is scheme-independent and the shift of $\mathrm{h}$ here is not in contradiction with the results above. Indeed, sending $\mathrm{h} \rightarrow \mathrm{h}+2$ leaves (3.34) invariant to 2 loops.

${ }^{24}$ Note that in the case when $S^{3}$ is interpreted as a coset $\mathrm{SO}(4) / \mathrm{SO}(3)$ the coupling of the symmetric space $\sigma$-model is given by $\mathrm{h}=2 R^{2}$ (cf. footnote 36 ).

${ }^{25} \mathrm{~A}$ similar approach may also be useful for clarifying the higher-loop deformation in abelian T-duality. In this case the model on the "tripled" configuration space can be found from the interpolating model for abelian T-duality. For example, consider the metric $\mathrm{G}=d y^{2}+a(y) d x^{2}$ and its classical dual $\tilde{\mathrm{G}}=d y^{2}+$ $a^{-1}(y) d \tilde{x}^{2}$ with the interpolating model given by $L=\left(\partial_{r} y\right)^{2}+a(y)\left(A_{r}\right)^{2}+\tilde{x} \epsilon^{r s} F_{r s}$ such that $y$ is a spectator coordinate. If we integrate out $\tilde{x}$ to give $A_{r}=\partial_{r} x$ we recover the original model for $x$. If we integrate out $A_{r}$ we find the T-dual model for $\tilde{x}$. If instead we set $A_{1}=\partial_{1} x$ and integrate out $A_{0}$ we get the "doubled" model of [73] for $x$ and $\tilde{x}$ (equivalent to the "axial" gauge choice in the appendix of [74]). The "tripled" model for $(x, \tilde{x}, \bar{x})$ is obtained by setting $A_{r}=\partial_{r} x+\epsilon_{r s} \partial^{s} \bar{x}: \mathcal{L}=\left(\partial_{r} y\right)^{2}+a(y)\left[\left(\partial_{r} x\right)^{2}-\left(\partial_{r} \bar{x}\right)^{2}+2 \epsilon^{r s} \partial_{r} x \partial_{s} \bar{x}\right]+\partial_{r} \tilde{x} \partial^{r} \bar{x}$. This may be interpreted as a $\sigma$-model on 4-dimensional target space with pp-wave metric and B-field.

${ }^{26}$ Here we consider only $\sigma$-models without potential terms. Adding potentials one can certainly arrange to have renormalizability in perturbation theory without having integrability. So, in general, integrability may imply renormalizability but not vice versa.
} 
well-known cases in which the classical integrability is anomalous, e.g. the bosonic $\mathbb{C} \mathbf{P}^{N}$ model $[75,76]$ (see also [77, 78]). However, this does not appear to be reflected in the 2-loop renormalizability. For the $\mathbb{C} \mathbf{P}^{N}$ model it has been conjectured that quantum integrability can be restored by including an additional free field [79] in the classical limit (related models have also appeared in $[80,81])$. The precise way in which this occurs and how it can be understood in the "tripled" configuration space remain to be understood. An alternative is to consider the supersymmetric $\mathbb{C} \mathbf{P}^{N} \sigma$-model in which there is no anomaly [82]. It could also prove insightful to redo the analysis in this paper for such models.

A potentially useful application of our results is to the $\eta$-model of $[36,37]$ and PoissonLie duality. Up to analytic continuation, the $\eta$-model and $\lambda$-model are related by limits and T-duality $[15,43]$ or by Poisson-Lie duality [15, 40-43]. These connections may be used to investigate both the renormalizability of the $\eta$-model and higher-loop corrections to non-abelian and Poisson-Lie duality. In our analysis of the $\lambda$-model we have computed the 2-loop $\beta$-function of the models (2.10) and (2.17). It would be interesting to extend this to the more general "doubly $\lambda$-deformed" models constructed in [51-54].

Another obvious generalization is to $\sigma$-models on supergroups and supercosets (see, e.g., $[29,58,83,84])$ where a generalization of our 2 -loop $\beta$-function expressions would, e.g., check the 2-loop finiteness of the model of [34].

\section{Acknowledgments}

We would like to thank R. Borsato, S. Demulder, F. Seibold, K. Sfetsos, D. Thompson and L. Wulff for useful discussions. We also thank K. Sfetsos for drawing our attention to ref. [53]. BH was supported by the Swiss National Science Foundation through the NCCR SwissMAP. NL was supported by the EPSRC grant EP/N509486/1. AAT was supported by the STFC grant ST/P000762/1.

\section{A Notation and conventions}

Our conventions for the non-zero components of the $2 \mathrm{~d}$ Minkowski metric $\eta_{r s}$ and the Levi-Civita symbol $\epsilon^{r s}$ are, respectively, $\eta_{00}=-\eta_{11}=-1$ and $\epsilon^{01}=-\epsilon^{10}=1$. The $2 \mathrm{~d}$ light-cone coordinates are defined as $\sigma^{ \pm}=\frac{1}{2}\left(\sigma^{0} \pm \sigma^{1}\right)$ so that $\partial_{ \pm}=\partial_{0} \pm \partial_{1}$. For $n$-forms we define components in the standard way: $\mathrm{H}_{(n)}=\frac{1}{n !} \mathrm{H}_{i_{1} \cdots i_{n}} d x^{i_{1}} \wedge \cdots \wedge d x^{i_{n}}$.

For an irreducible finite-dimensional representation of the compact simple Lie group $G$ we normalize the generators $\left\{T^{a}\right\}$ and the invariant bilinear form such that ${ }^{27}$

$$
\left[T^{a}, T^{b}\right]=f_{c}^{a b} T^{c}, \quad \operatorname{Tr}\left(T^{a} T^{b}\right)=\delta^{a b}, \quad f^{a b} f_{a b d}=-2 c_{G} \delta_{c d}, \quad c_{G} \equiv c_{2}(G),
$$

where $c_{2}(G)$ is the dual Coxeter number of the group $G$ and indices are raised and lowered with $\delta^{a b}$ and its inverse. This implies that Tr is related to the usual matrix trace, tr, by

$$
\operatorname{Tr}=\frac{1}{2 \chi_{G, R}} \operatorname{tr},
$$

\footnotetext{
${ }^{27}$ This means that we have hermitian generators and imaginary structure constants.
} 
where $\chi_{G, R}$ is the index of the representation. ${ }^{28}$ It then follows that

$$
f_{a}^{d e} f_{b e}^{f} f_{c d f}=c_{G} f_{a b c} .
$$

In the coset case, $G / H$ is assumed to be a compact irreducible symmetric space. Introducing the orthogonal splitting $\left\{T^{a}\right\}=\left\{T^{\alpha}, T^{i}\right\}$, where $\left\{T^{\alpha}\right\}$ are the generators of $H$, we have $\operatorname{Tr}\left(T^{\alpha} T^{i}\right)=0$ and the non-zero commutation relations are given by

$$
\left[T^{\alpha}, T^{\beta}\right]=f_{\gamma}^{\alpha \beta} T^{\gamma}, \quad\left[T^{\alpha}, T^{i}\right]=f^{\alpha i}{ }_{j} T^{j}, \quad\left[T^{i}, T^{j}\right]=f^{i j}{ }_{\alpha} T^{\alpha} .
$$

In the computation of the 2-loop RG flow we make use of the identities

$$
\begin{aligned}
f^{\alpha i}{ }_{j} f^{\beta j}{ }_{i} & =2 \chi_{H, G / H} \delta^{\alpha \beta}, \quad f^{\alpha k}{ }_{i} f_{\alpha k j}=-c_{G} \delta_{i j}, \\
f^{\alpha m l} f^{\beta k}{ }_{l} f_{\alpha k i} f_{\beta m j} & =-\frac{1}{2} f^{\alpha m}{ }_{l} f^{\beta l}{ }_{m} f_{\alpha}{ }^{k}{ }_{i} f_{\beta k j}=c_{G}\left(c_{G}-c_{H}\right) \delta_{i j}, \quad c_{H} \equiv c_{G}-\chi_{H, G / H},
\end{aligned}
$$

where $\chi_{H, G / H}$ is the index of the representation of $\operatorname{Lie}(H)$ in which the coset directions transform, i.e. of the matrices $\left(f^{\alpha}\right)^{i}{ }_{j}=f^{\alpha i}{ }_{j}$. When both $G$ and $H$ are simple we have that $c_{H} \equiv \frac{\chi_{G, R}}{\chi_{H, R}} c_{2}(H)$ where $c_{2}(H)$ is the dual Coxeter number of the group $H$ and $\chi_{H, R}$ is the index of the representation $\left\{T^{\alpha}\right\}$ of $\operatorname{Lie}(H)$. For more general subgroups $H$ the constant $c_{H}$ takes a more complicated form. In the case of type II symmetric spaces, i.e. $\frac{H \times H}{H}$, we have $c_{G}=c_{2}(H)$ and $c_{H}=\frac{1}{2} c_{2}(H)$. Finally, the expressions for the dual non-compact irreducible Riemannian symmetric space can be found by the formal substitution $k \rightarrow-k{ }^{29}$

\section{B 2-loop $\beta$-function of squashed PCM and $G / H$ coset $\sigma$-models}

Let $G$ and $H \subset G$ be compact simple Lie groups and $G / H$ be a compact irreducible symmetric space. Below we shall consider the renormalisation of the "squashed" PCM model with action $\mathcal{S}=\frac{1}{4 \pi} \int d^{2} \sigma \mathcal{L}$ where ${ }^{30}$

$$
\begin{aligned}
\mathcal{L} & =-\frac{1}{2} \mathrm{~h}\left(J_{+}^{i} J_{-}^{i}+\varepsilon J_{+}^{\alpha} J_{-}^{\alpha}\right)=-\frac{1}{2}\left(\mathrm{~h} J_{+}^{i} J_{-}^{i}+\widetilde{\mathrm{h}} J_{+}^{\alpha} J_{-}^{\alpha}\right), \\
J_{ \pm}^{a} & =\left(J_{ \pm}^{\alpha}, J_{ \pm}^{i}\right)=\operatorname{Tr}\left(T^{a} g^{-1} \partial_{ \pm} g\right), \quad \widetilde{\mathrm{h}} \equiv \mathrm{h} \varepsilon .
\end{aligned}
$$

\footnotetext{
${ }^{28}$ We use a somewhat unconventional normalization of the generators and thus the structure constants (by a factor of $\sqrt{2}$ ) compared to the standard relations $\operatorname{tr}\left(T^{\prime a} T^{\prime b}\right)=\chi_{G, R} \delta^{a b}$ and $f^{\prime a b} f_{a b d}^{\prime}=-c_{G} \delta_{c d}$. Nevertheless, our normalizations are consistent with the standard values for the indices of representations. For the fundamental representation we have $\chi_{\mathrm{SU}(N) \text {,fund }}=\chi_{\mathrm{Sp}(N) \text {,fund }}=\frac{1}{2}$ and (for $\left.N \geq 5\right) \chi_{\mathrm{SO}(N) \text {,fund }}=1$, while for the adjoint representation the index is equal to the dual Coxeter number: $\chi_{\mathrm{SU}(N) \text {,adj }}=c_{2}(\mathrm{SU}(N))=$ $N, \chi_{\mathrm{Sp}(N), \mathrm{adj}}=c_{2}(\operatorname{Sp}(N))=N+1$ and $($ for $N \geq 5) \chi_{\mathrm{SO}(N), \mathrm{adj}}=c_{2}(\mathrm{SO}(N))=N-2$.

${ }^{29}$ In the classification of irreducible Riemannian symmetric spaces, excluding the special case of flat space, every compact space has a corresponding non-compact space, often referred to as a duality. The simplest example of this is the sphere and the hyperboloid. The non-compact irreducible Riemannian symmetric spaces take the form $G / H$ with $H$ the maximal compact subgroup of $G$. Therefore, the coset directions are all non-compact and for a positive-definite signature of the metric we replace $k \rightarrow-k$ compared to the compact case.

${ }^{30}$ As in appendix A (cf. (A.4)) we denote the $H$ and $G / H$ algebra indices by $\alpha$ and $i$ respectively, i.e. the $G$ algebra index is $a=\{\alpha, i\}$. The overall minus sign is due to the conventions explained in appendix A, in particular the choice to use hermitian generators $T^{a}$ satisfying (A.1). The coupling $\mathrm{h}$ is related to the conventional PCM coupling $\mathrm{g}$ by $\mathrm{h}=\frac{2}{\mathrm{~g}^{2}}$. Indices in (B.1) and (B.4) are contracted with $\delta_{i j}$ and $\delta_{\alpha \beta}$.
} 
It interpolates between the PCM on the group $G(\varepsilon=1)$ and the $G / H$ symmetric space $\sigma$-model $(\varepsilon \rightarrow 0)$. Also, in the limit

$$
\mathrm{h} \rightarrow \infty, \quad \varepsilon \rightarrow 0, \quad \widetilde{\mathrm{h}}=\text { fixed }
$$

when the coset part decouples, the model (B.1) reduces to the PCM on the group $H$ with the coupling $\widetilde{\mathrm{h}}$.

For $\varepsilon \neq 0$, the action (B.1) has global $G \times H$ symmetry: $g \rightarrow u g v, u \in G, v \in H$. For the symmetric space $\sigma$-model case of $\varepsilon=0$, the global $H$ symmetry is enhanced to a gauge symmetry. Due to these symmetries ${ }^{31}$ the model (B.1) is renormalizable with only the two couplings h and $\varepsilon$ (or $\widetilde{\mathrm{h}}$ ) running. This will be explicitly verified below in the 2-loop approximation (expanding in large $\mathrm{h}$ for fixed $\varepsilon$ ).

We define the target space vielbein $E^{a} \equiv E_{\mu}^{a} d x^{\mu}=\left(J^{i}, J^{\alpha}\right)$ where $J^{a}$ are 1-forms corresponding to the currents in (B.1) (cf. (2.19), (2.32)), so that the corresponding metric of the $\sigma$-model in (1.11) takes the form

$$
\mathrm{G}_{\mu \nu} d x^{\mu} d x^{\nu}=-\frac{1}{2} \mathrm{~h}\left(J^{i} J^{i}+\varepsilon J^{\alpha} J^{\alpha}\right) .
$$

The spin connection $\omega^{a}{ }_{b}$ is found to have the following components

$$
\omega^{\alpha}{ }_{\beta}=-\frac{1}{2} f^{\alpha}{ }_{\beta \gamma} J^{\gamma}, \quad \omega^{\alpha}{ }_{i}=-\frac{1}{2} f^{\alpha}{ }_{i j} J^{j}, \quad \omega^{i}{ }_{j}=-\left(1-\frac{1}{2} \varepsilon\right) f_{j \alpha}^{i} J^{\alpha},
$$

and the non-zero components of the corresponding Riemann tensor are given by (up to permutations and symmetries)

$$
\begin{aligned}
R^{\alpha}{ }_{\beta \delta \varepsilon} & =\frac{1}{4} f_{\beta c}^{\alpha} f_{\delta \varepsilon}^{c}, & R^{\alpha}{ }_{i \gamma k} & =\frac{1}{4}\left(f_{k c}^{i} f^{c}{ }_{\alpha \gamma}-\varepsilon f_{k c}^{\alpha} f_{i \gamma}^{c}\right), \\
R_{j \beta \gamma}^{i} & =\frac{1}{2} \varepsilon\left(1-\frac{1}{2} \varepsilon\right) f_{j c}^{i} f_{\beta \gamma}^{c}, & R^{i}{ }_{j k l} & =\left(1-\frac{3}{4} \varepsilon\right) f_{j c}^{i} f_{k l}^{c} .
\end{aligned}
$$

In the PCM case of $\varepsilon=1$, the curvature (B.6) reduces to the standard group space expression

$$
R_{b d e}^{a}=\frac{1}{4} f_{b c}^{a} f_{d e}^{c} .
$$

For $\mathrm{B}_{\mu \nu}=0$ the 2-loop RG equation (1.12) becomes the familiar one $[21]^{32}$

$$
\frac{d \mathrm{G}_{\mu \nu}}{d t}=R_{\mu \nu}+\frac{1}{2} R_{\mu}^{\lambda \rho \sigma} R_{\nu \lambda \rho \sigma}
$$

\footnotetext{
${ }^{31}$ This follows from the assumption that $H$ is simple and $G / H$ is an irreducible symmetric space. Therefore both $\left\{T^{\alpha}\right\}$ and $\left\{T^{i}\right\}$ transform in irreducible representations of $\operatorname{Lie}(H)$ and the two terms in (B.1) are the only ones that respect the global $G \times H$ symmetry of the model. In principle, this argument applies to any coset space $G / H$ for which this irreducibility of representations holds. At the $\varepsilon=0$ point we recover the symmetric space or coset $\sigma$-model and the $H$ gauge symmetry implies that just the irreducibility of $\left\{T^{i}\right\}$ is sufficient for renormalizability. An additional subtlety can occur when the irreducible representations are reducible over $\mathbb{C}$. Then it may be possible to construct new terms respecting the symmetries invoked above (cf. footnote 9). We expect any such new terms to be excluded by additional symmetries such as parity.

${ }^{32}$ Here we ignore the diffeomorphism term which is not allowed by the global $G \times H$ symmetry.
} 
Substituting in (B.6), one finds that the model (B.1) is 2-loop renormalizable with $\mathrm{h}$ and $\varepsilon$ running according to ${ }^{33}$

$$
\begin{aligned}
\frac{d}{d t} \mathrm{~h}=(2-\varepsilon) c_{G}+ & \frac{1}{2} c_{G} \mathrm{~h}^{-1}\left[c_{G}\left(8-12 \varepsilon+5 \varepsilon^{2}\right)-c_{H}(1-\varepsilon)(5-4 \varepsilon)\right] \\
\frac{d}{d t} \varepsilon=-\mathrm{h}^{-1}(1-\varepsilon) & \left(2 c_{G} \varepsilon-(1+\varepsilon) c_{H}\right. \\
& \left.+\frac{1}{2} \mathrm{~h}^{-1}\left[4 c_{G}^{2} \varepsilon(2-\varepsilon)-c_{G} c_{H} \varepsilon(11-4 \varepsilon)-c_{H}^{2}\left(\varepsilon^{-1}+1-5 \varepsilon+\varepsilon^{2}\right)\right]\right)
\end{aligned}
$$

Thus $\varepsilon=1$ is a fixed line of (B.10), on which (B.9) reduces to the 2 -loop $\beta$-function of the PCM on the group $G$

$$
\frac{d}{d t} \mathrm{~h}=c_{G}+\frac{1}{2} c_{G}^{2} \mathrm{~h}^{-1} .
$$

The same expression was found from the NAD limit of the $\lambda$-model in (2.31).

In the special case of the squashed 3-sphere or squashed SU(2) PCM (with $H=\mathrm{U}(1)$, i.e. $\left.c_{G}=2, c_{H}=0\right)$ the expressions in (B.9), (B.10) agree with the 2-loop $\beta$-functions found in [20]: the couplings $\lambda$ and $g$ used there are related to ours by $c_{G} \mathrm{~h}^{-1}=\frac{\lambda}{4 \pi}, \varepsilon=1+g$.

Written in terms of the couplings $h$ and $\widetilde{h}$ in (B.1) the equations (B.9), (B.10) take the form

$$
\begin{aligned}
& \frac{d}{d t} \mathrm{~h}=2 c_{G}+\frac{1}{2} c_{G}\left(8 c_{G}-5 c_{H}-2 \widetilde{\mathrm{h}}\right) \mathrm{h}^{-1}-\frac{3}{2} c_{G}\left(4 c_{G}-3 c_{H}\right) \widetilde{\mathrm{h}} \mathrm{h}^{-2}+\frac{1}{2} c_{G}\left(5 c_{G}-4 c_{H}\right) \widetilde{\mathrm{h}}^{2} \mathrm{~h}^{-3}, \\
& \frac{d}{d t} \widetilde{\mathrm{h}}=c_{H}+\frac{1}{2} c_{H}^{2} \widetilde{\mathrm{h}}^{-1}+\left(c_{G}-c_{H}\right) \widetilde{\mathrm{h}} \mathrm{h}^{-2}\left[3 c_{H}+\widetilde{\mathrm{h}}-3 c_{H} \widetilde{\mathrm{h}} \mathrm{h}^{-1}+\frac{1}{2}\left(c_{G}+c_{H}\right) \widetilde{\mathrm{h}}^{2} \mathrm{~h}^{-2}\right] .
\end{aligned}
$$

These two equations become the same and equal to (B.11) at the PCM fixed point $h=\widetilde{h}$ $(\varepsilon=1)$. In the limit (B.3) when $\mathrm{h}^{-1} \rightarrow 0$ we get from (B.13) the correct 2-loop RG equation for the coupling $\widetilde{\mathrm{h}}$ of the PCM on the group $H$ (cf. (B.11) $)^{34}$

$$
\frac{d}{d t} \widetilde{\mathrm{h}}=c_{H}+\frac{1}{2} c_{H}^{2} \widetilde{\mathrm{h}}^{-1}
$$

Let us now consider the coset space limit $\epsilon=0$ (or $\widetilde{\mathrm{h}}=0$ ). In the abelian $H$ case when $c_{H}=0$, we have $\varepsilon=0$ solving (B.10) while (B.9) reduces to $\frac{d}{d t} \mathrm{~h}=2 c_{G}+4 c_{G}^{2} \mathrm{~h}^{-1}$, which agrees with the symmetric space $\sigma$-model $\beta$-function in (2.40), (B.16). However, for non-abelian $H$ with $c_{H} \neq 0$, the $\varepsilon \rightarrow 0$ limit of (B.10) is singular. The limit of (B.9), while giving the correct 1-loop part of the $\beta$-function for the $G / H$ symmetric space $\sigma$-model (see, e.g., [58]), fails to do so at the 2-loop order. Indeed this limit is subtle and should be treated separately due to the $H$ gauge symmetry that arises in the model (B.1) at the point $\varepsilon=0$.

This gauge symmetry is reflected in the degeneracy of the metric (B.4) in the $\varepsilon \rightarrow 0$ limit. One option is to set $\varepsilon=0$ and then use the standard gauge fixing procedure. For

\footnotetext{
${ }^{33}$ Note that these equations depend on $\mathrm{h}, c_{G}$ and $c_{H}$ only through the ratios $c_{G} \mathrm{~h}^{-1}$ and $\frac{c_{H}}{c_{G}}$.

${ }^{34}$ Expressed in terms of the physical coupling $\mathrm{h}^{-1}$ eq. (B.12) is $\frac{d}{d t} \mathrm{~h}^{-1}=-2 c_{G} \mathrm{~h}^{-2}+\ldots$ so $\mathrm{h}^{-1}=0$ is (trivially) a fixed line.
} 
example, we may fix an analog of "axial" gauge $N^{r} J_{r}^{\alpha}=f^{\alpha}(\sigma)$ where $N^{r}$ is a constant $2 \mathrm{~d}$ vector. Then averaging over $f$ with exponential weight $\sim u f^{\alpha} f^{\alpha}$ will give an extra gaugefixing term $u\left(N^{r} J_{r}^{\alpha}\right)^{2}$ in the coset $\sigma$-model action. The resulting on-shell effective action and thus the on-shell UV divergences should not depend on the value of the gauge-fixing parameter $u$ or the choice of $N^{r}$. As this procedure is somewhat cumbersome, we may try to use a short-cut.

Indeed, observing that averaging over $N^{r}$ should effectively restore $2 \mathrm{~d}$ Lorentz invariance in gauge-invariant expressions we may simply add $u\left(J_{r}^{\alpha}\right)^{2}$ or, equivalently, go back to (B.1) with $u=\varepsilon$. This may be viewed as using $\varepsilon \ll 1$ as a regulator, breaking the gauge invariance and lifting the degeneracy. Then after computing the Riemann tensor, we will need to project out the components in the degenerate $H$ (or $\alpha$ ) directions and finally take the $\varepsilon \rightarrow 0$ limit and compute the $\beta$-function. ${ }^{35}$ Projecting out the $\alpha$ components of (B.6) and setting $\varepsilon=0$ gives the standard expression for the symmetric space Riemann tensor (see, e.g., [85-87])

$$
R_{j k l}^{i}=f_{j \alpha}^{i} f_{k l}^{\alpha} .
$$

An alternative approach (equivalent to explicitly solving the gauge condition rather than adding it to the action to lift the degeneracy) would be to take $g$ to be parametrized by the $\operatorname{dim} G-\operatorname{dim} H$ physical degrees of freedom. The particular parametrization is not important, but one could take, e.g., $g=\exp \left(i v_{i} T^{i}+i v_{\alpha}\left(v_{i}\right) T^{\alpha}\right)$. Then we may expand $J^{\alpha}$ in the vielbein $E^{i}=J^{i}$, i.e. $J^{\alpha}=F_{i}^{\alpha}(g) E^{i}$. Computing the spin correction and the corresponding curvature, the latter does not depend on $F_{i}^{\alpha}(g)$, as expected by gauge invariance, and agrees with (B.15).

Plugging (B.15) into the RG equation (B.8) we then obtain the 2-loop $\beta$-function for the symmetric space $\sigma$-model

$$
\frac{d}{d t} \mathrm{~h}=2 c_{G}+4 c_{G}\left(c_{G}-c_{H}\right) \mathrm{h}^{-1}
$$

This expression agrees with previous results found for particular cosets in [21, 62, 65-67]. ${ }^{36}$ It also matches the result found from the NAD limit of the $\lambda$-model in (2.40). In contrast to (B.9), (B.10), the expression for the $\beta$-function for $\mathrm{h}$ in (B.16) is valid for any compact irreducible symmetric space (cf. footnote 31 ) with $c_{G}$ and $c_{H}$ defined in appendix A, i.e. $G$ and $H$ need not be simple. ${ }^{37}$

\footnotetext{
${ }^{35}$ In a systematic gauge-fixing the analog of $\varepsilon$ or the gauge-fixing parameter $u$ should automatically disappear from the on-shell divergences. Note also that this procedure is effectively equivalent to fixing a "transverse" gauge in which the $H$-components of the quantum fields are set to zero so the curvature tensor coefficients in the $\sigma$-model vertices are contracted with the propagators containing projectors to $G / H$. In addition, the classical fields (in the background field method for computing divergences) have only $G / H$ components due to the classical gauge invariance in the $\varepsilon=0$ limit.

${ }^{36}$ For example, in the case of the sphere $S^{N-1}=\mathrm{SO}(N) / \mathrm{SO}(N-1)$, using the fundamental representation of $\mathrm{SO}(N)$ and following the notation in appendix A we have $c_{G}=N-2, c_{H}=N-3, \chi_{G \text {,fund }}=\chi_{H \text {,fund }}=1$. Normalizing the Lagrangian as in (1.10), (1.11) we find that $\mathrm{h}=2 R^{2}$ where $R$ is the radius of the sphere. Therefore, from (B.16) we find the standard 2-loop RG equation for $S^{N-1}: \frac{d}{d t} R^{2}=(N-2)+(N-2) R^{-2}$.

${ }^{37}$ Let us note for completeness that renormalizability of $\sigma$-models on some homogeneous cosets (not necessarily symmetric spaces) was discussed, e.g., in [88-90].
} 
Let us note that since (B.1) is a two-coupling theory, the 2-loop (and higher) terms in the $\beta$-functions (B.9), (B.10) are not, in general, invariant under scheme changes or redefinitions of the couplings $\mathrm{h}$ and $\varepsilon$. However, they still contain some invariant information as the limits $\varepsilon=1$ (PCM on $G)$, (B.3) (PCM on $H$ ) and $\varepsilon=0(G / H$ coset space) lead to one-coupling models whose 2-loop $\beta$-functions are invariant under coupling redefinitions.

We finish with a curious observation that the 2-loop $\beta$-functions (B.9), (B.10) vanish if

$$
\varepsilon=2, \quad c_{G}=\frac{3}{4} c_{H} .
$$

Indeed, if there are such $G$ and $H$ that the relation $c_{G}=\frac{3}{4} c_{H}$ can be satisfied, then the expressions in (B.9), (B.10) simplify to

$$
\begin{aligned}
\frac{d}{d t} \mathrm{~h} & =(2-\varepsilon) c_{G}\left[1+\frac{1}{6} c_{G} \mathrm{~h}^{-1}(2+\varepsilon)\right], \\
\frac{d}{d t} \varepsilon & =\frac{2}{3} \mathrm{~h}^{-1}(2-\varepsilon)(1-\varepsilon) c_{G}\left[1+\frac{1}{3} c_{G} \mathrm{~h}^{-1}\left(2 \varepsilon^{-1}+3-\varepsilon\right)\right],
\end{aligned}
$$

and thus $\varepsilon=2$ is a 2-loop fixed point. This suggests that the corresponding squashed PCM with $\varepsilon=2$ is an exact CFT for any value of h. Therefore, it is a particularly interesting representation theory question as to whether there are solutions to the condition $c_{G}=\frac{3}{4} c_{H}$. For this it may be necessary to consider supergroups.

Open Access. This article is distributed under the terms of the Creative Commons Attribution License (CC-BY 4.0), which permits any use, distribution and reproduction in any medium, provided the original author(s) and source are credited.

\section{References}

[1] V.A. Fateev, E. Onofri and A.B. Zamolodchikov, Integrable deformations of $O(3)$ sigma model. The sausage model, Nucl. Phys. B 406 (1993) 521 [INSPIRE].

[2] V.A. Fateev, The sigma model (dual) representation for a two-parameter family of integrable quantum field theories, Nucl. Phys. B 473 (1996) 509 [INSPIRE].

[3] S.L. Lukyanov, The integrable harmonic map problem versus Ricci flow, Nucl. Phys. B $\mathbf{8 6 5}$ (2012) 308 [arXiv: 1205.3201] [INSPIRE].

[4] V. Fateev, Classical and quantum integrable sigma models. Ricci flow, "nice duality" and perturbed rational conformal field theories, arXiv:1902.02811 [INSPIRE].

[5] V.A. Fateev and A.V. Litvinov, Integrability, Duality and Sigma Models, JHEP 11 (2018) 204 [arXiv: 1804.03399] [INSPIRE].

[6] A.V. Litvinov and L.A. Spodyneiko, On dual description of the deformed $O(N)$ sigma model, JHEP 11 (2018) 139 [arXiv:1804.07084] [INSPIRE].

[7] B. Hoare, N. Levine and A.A. Tseytlin, Integrable 2d sigma models: quantum corrections to geometry from RG flow, Nucl. Phys. B 949 (2019) 114798 [arXiv: 1907.04737] [INSPIRE].

[8] K. Sfetsos, Integrable interpolations: From exact CFTs to non-abelian T-duals, Nucl. Phys. B 880 (2014) 225 [arXiv:1312.4560] [INSPIRE]. 
[9] T.J. Hollowood, J.L. Miramontes and D.M. Schmidtt, Integrable Deformations of Strings on Symmetric Spaces, JHEP 11 (2014) 009 [arXiv:1407.2840] [INSPIRE].

[10] K. Sfetsos and A.A. Tseytlin, Chiral gauged WZNW models and heterotic string backgrounds, Nucl. Phys. B 415 (1994) 116 [hep-th/9308018] [INSPIRE].

[11] K. Sfetsos and A.A. Tseytlin, Antisymmetric tensor coupling and conformal invariance in sigma models corresponding to gauged WZNW theories, Phys. Rev. D 49 (1994) 2933 [hep-th/9310159] [INSPIRE].

[12] A.A. Tseytlin, On a 'universal' class of WZW type conformal models, Nucl. Phys. B 418 (1994) 173 [hep-th/9311062] [INSPIRE].

[13] S.-w. Chung and S.H.H. Tye, Chiral gauged WZW theories and coset models in conformal field theory, Phys. Rev. D 47 (1993) 4546 [hep-th/9202002] [inSPIRE].

[14] G. Itsios, K. Sfetsos and K. Siampos, The all-loop non-abelian Thirring model and its $R G$ flow, Phys. Lett. B 733 (2014) 265 [arXiv:1404.3748] [INSPIRE].

[15] B. Hoare and A.A. Tseytlin, On integrable deformations of superstring sigma models related to $A d S_{n} \times S^{n}$ supercosets, Nucl. Phys. B 897 (2015) 448 [arXiv:1504.07213] [INSPIRE].

[16] D. Kutasov, Duality Off the Critical Point in Two-dimensional Systems With Non-abelian Symmetries, Phys. Lett. B 233 (1989) 369 [INSPIRE].

[17] A. Subbotin and I.V. Tyutin, On the equivalence of dual theories, Int. J. Mod. Phys. A 11 (1996) 1315 [Erratum ibid. A 11 (1996) 2231] [hep-th/9506132] [INSPIRE].

[18] L.K. Balazs, J. Balog, P. Forgacs, N. Mohammedi, L. Palla and J. Schnittger, Quantum equivalence of sigma models related by non-abelian duality transformations, Phys. Rev. D 57 (1998) 3585 [hep-th/9704137] [INSPIRE].

[19] G. Bonneau and P.-Y. Casteill, Dualized sigma models at the two loop order, Nucl. Phys. B 607 (2001) 293 [hep-th/0103260] [INSPIRE].

[20] J. Balog, P. Forgacs, Z. Horvath and L. Palla, Perturbative quantum (in)equivalence of dual sigma models in two-dimensions, Nucl. Phys. Proc. Suppl. 49 (1996) 16 [hep-th/9601091] [INSPIRE].

[21] D.H. Friedan, Nonlinear Models in Two + Epsilon Dimensions, Annals Phys. 163 (1985) 318 [INSPIRE].

[22] E. Braaten, T.L. Curtright and C.K. Zachos, Torsion and Geometrostasis in Nonlinear Sigma Models, Nucl. Phys. B 260 (1985) 630 [Erratum ibid. B 266 (1986) 748] [InSPIRE].

[23] R.R. Metsaev and A.A. Tseytlin, Order alpha-prime (two loop) equivalence of the string equations of motion and the sigma model Weyl invariance conditions: dependence on the dilaton and the antisymmetric tensor, Nucl. Phys. B 293 (1987) 385 [INSPIRE].

[24] R.R. Metsaev and A.A. Tseytlin, Two loop beta function for the generalized bosonic sigma model, Phys. Lett. B 191 (1987) 354 [INSPIRE].

[25] C.M. Hull and P.K. Townsend, The Two Loop Beta Function for $\sigma$ Models With Torsion, Phys. Lett. B 191 (1987) 115 [INSPIRE].

[26] D. Zanon, Two Loop Beta Functions and Low-energy String Effective Action for the Two-dimensional Bosonic Nonlinear $\sigma$ Model With a Wess-Zumino-Witten Term, Phys. Lett. B 191 (1987) 363 [INSPIRE]. 
[27] N. Beisert et al., Review of AdS/CFT Integrability: An Overview, Lett. Math. Phys. 99 (2012) 3 [arXiv: 1012.3982] [INSPIRE].

[28] N. Gromov, V. Kazakov, S. Leurent and D. Volin, Quantum Spectral Curve for Planar $\mathcal{N}=4$ Super-Yang-Mills Theory, Phys. Rev. Lett. 112 (2014) 011602 [arXiv:1305.1939] [INSPIRE].

[29] K. Zarembo, Strings on Semisymmetric Superspaces, JHEP 05 (2010) 002 [arXiv: 1003.0465] [INSPIRE].

[30] L. Wulff, Superisometries and integrability of superstrings, JHEP 05 (2014) 115 [arXiv: 1402.3122] [INSPIRE].

[31] O. Lunin and J.M. Maldacena, Deforming field theories with $\mathrm{U}(1) \times \mathrm{U}(1)$ global symmetry and their gravity duals, JHEP 05 (2005) 033 [hep-th/0502086] [INSPIRE].

[32] S.A. Frolov, R. Roiban and A.A. Tseytlin, Gauge-string duality for superconformal deformations of $\mathcal{N}=4$ super Yang-Mills theory, JHEP 07 (2005) 045 [hep-th/0503192] [INSPIRE].

[33] S. Frolov, Lax pair for strings in Lunin-Maldacena background, JHEP 05 (2005) 069 [hep-th/0503201] [INSPIRE].

[34] T.J. Hollowood, J.L. Miramontes and D.M. Schmidtt, An Integrable Deformation of the $A d S_{5} \times S^{5}$ Superstring, J. Phys. A 47 (2014) 495402 [arXiv:1409.1538] [inSPIRE].

[35] F. Delduc, M. Magro and B. Vicedo, An integrable deformation of the $A d S_{5} \times S^{5}$ superstring action, Phys. Rev. Lett. 112 (2014) 051601 [arXiv: 1309.5850] [INSPIRE].

[36] C. Klimčík, Yang-Baxter sigma models and dS/AdS T duality, JHEP 12 (2002) 051 [hep-th/0210095] [INSPIRE].

[37] F. Delduc, M. Magro and B. Vicedo, On classical q-deformations of integrable sigma-models, JHEP 11 (2013) 192 [arXiv: 1308.3581] [INSPIRE].

[38] C. Klimčík and P. Ševera, Dual non-Abelian duality and the Drinfeld double, Phys. Lett. B 351 (1995) 455 [hep-th/9502122] [INSPIRE].

[39] C. Klimčík, Poisson-Lie T-duality, Nucl. Phys. Proc. Suppl. 46 (1996) 116 [hep-th/9509095] [INSPIRE].

[40] B. Vicedo, Deformed integrable $\sigma$-models, classical R-matrices and classical exchange algebra on Drinfel'd doubles, J. Phys. A 48 (2015) 355203 [arXiv:1504.06303] [InSPIRE].

[41] K. Sfetsos, K. Siampos and D.C. Thompson, Generalised integrable $\lambda$ - and $\eta$-deformations and their relation, Nucl. Phys. B 899 (2015) 489 [arXiv: 1506.05784] [INSPIRE].

[42] C. Klimčík, $\eta$ and $\lambda$ deformations as E-models, Nucl. Phys. B 900 (2015) 259 [arXiv: 1508.05832] [INSPIRE].

[43] B. Hoare and F.K. Seibold, Poisson-Lie duals of the $\eta$ deformed symmetric space sigma model, JHEP 11 (2017) 014 [arXiv: 1709.01448] [INSPIRE].

[44] R. Borsato and L. Wulff, Target space supergeometry of $\eta$ and $\lambda$-deformed strings, JHEP 10 (2016) 045 [arXiv: 1608.03570] [INSPIRE].

[45] B. Hoare and F.K. Seibold, Supergravity backgrounds of the $\eta$-deformed $A d S_{2} \times S^{2} \times T^{6}$ and $A d S_{5} \times S^{5}$ superstrings, JHEP 01 (2019) 125 [arXiv: 1811.07841] [INSPIRE].

[46] A.M. Polyakov and P.B. Wiegmann, Theory of Nonabelian Goldstone Bosons, Phys. Lett. 131B (1983) 121 [INSPIRE]. 
[47] A.M. Polyakov, Two-dimensional quantum gravity: Superconductivity at high $T_{c}$, in: Fields, Strings and Critical Phenomena, Proc. of Les Houches 1988, eds.: E. Brézin and J. Zinn-Justin, North-Holland (1990).

[48] E. Guadagnini, M. Martellini and M. Mintchev, Scale Invariance Sigma Models On Homogeneous Spaces, Phys. Lett. B 194 (1987) 69 [InSPIRE].

[49] O.A. Solovev, Towards conversion of the space of Thirring models into the model space for groups, Phys. Lett. B 309 (1993) 275 [INSPIRE].

[50] C.M. Hull and O.A. Solovev, Conformal points and duality of non-abelian Thirring Models and interacting WZNW models, Nucl. Phys. B 459 (1996) 243 [hep-th/9503021] [INSPIRE].

[51] G. Georgiou and K. Sfetsos, A new class of integrable deformations of CFTs, JHEP 03 (2017) 083 [arXiv : 1612.05012] [INSPIRE].

[52] G. Georgiou, E. Sagkrioti, K. Sfetsos and K. Siampos, Quantum aspects of doubly deformed CFTs, Nucl. Phys. B 919 (2017) 504 [arXiv:1703.00462] [InSPIRE].

[53] G. Georgiou, K. Sfetsos and K. Siampos, Double and cyclic $\lambda$-deformations and their canonical equivalents, Phys. Lett. B 771 (2017) 576 [arXiv: 1704.07834] [INSPIRE].

[54] G. Georgiou and K. Sfetsos, Integrable flows between exact CFTs, JHEP 11 (2017) 078 [arXiv: 1707.05149] [INSPIRE].

[55] B. Gerganov, A. LeClair and M. Moriconi, On the beta function for anisotropic current interactions in 2-D, Phys. Rev. Lett. 86 (2001) 4753 [hep-th/0011189] [INSPIRE].

[56] A. LeClair, Chiral stabilization of the renormalization group for flavor and color anisotropic current interactions, Phys. Lett. B 519 (2001) 183 [hep-th/0105092] [INSPIRE].

[57] K. Sfetsos and K. Siampos, Gauged WZW-type theories and the all-loop anisotropic non-abelian Thirring model, Nucl. Phys. B 885 (2014) 583 [arXiv:1405.7803] [INSPIRE].

[58] C. Appadu and T.J. Hollowood, Beta function of $k$ deformed $A d S_{5} \times S^{5}$ string theory, JHEP 11 (2015) 095 [arXiv: 1507.05420] [INSPIRE].

[59] E. Witten, Non-abelian Bosonization in Two Dimensions, Commun. Math. Phys. 92 (1984) 455 [INSPIRE].

[60] M. Bos, Dimensional Regularization in the Wess-Zumino-Witten Model, Phys. Lett. B 189 (1987) 435 [INSPIRE].

[61] A. McKane and M. Stone, Nonlinear sigma models: a perturbative approach to symmetry restoration, Nucl. Phys. B 163 (1980) 169 [InSPIRE].

[62] S. Hikami, Three Loop Beta-Functions of Nonlinear Sigma Models on Symmetric Spaces, Phys. Lett. 98B (1981) 208 [INSPIRE].

[63] B.E. Fridling and A. Jevicki, Dual Representations and Ultraviolet Divergences in Nonlinear $\sigma$ Models, Phys. Lett. 134B (1984) 70 [inSPIRE].

[64] E.S. Fradkin and A.A. Tseytlin, Quantum Equivalence of Dual Field Theories, Annals Phys. 162 (1985) 31 [INSPIRE].

[65] E. Brézin and J. Zinn-Justin, Renormalization of the nonlinear $\sigma$ model in $2+\epsilon$ dimensions. Application to the Heisenberg ferromagnets, Phys. Rev. Lett. 36 (1976) 691 [INSPIRE].

[66] S. Hikami and E. Brézin, Three Loop Calculations in the Two-Dimensional Nonlinear Sigma Model, J. Phys. A 11 (1978) 1141 [INSPIRE]. 
[67] E. Brézin, S. Hikami and J. Zinn-Justin, Generalized Nonlinear $\sigma$ Models With Gauge Invariance, Nucl. Phys. B 165 (1980) 528 [INSPIRE].

[68] A.S. Schwarz and A.A. Tseytlin, Dilaton shift under duality and torsion of elliptic complex, Nucl. Phys. B 399 (1993) 691 [hep-th/9210015] [InSPIRE].

[69] I. Kawaguchi and K. Yoshida, Hidden Yangian symmetry in sigma model on squashed sphere, JHEP 11 (2010) 032 [arXiv: 1008.0776] [INSPIRE].

[70] N. Kaloper and K.A. Meissner, Duality beyond the first loop, Phys. Rev. D 56 (1997) 7940 [hep-th/9705193] [INSPIRE].

[71] S. Parsons, T duality and conformal invariance at two loops, Phys. Rev. D 61 (2000) 086002 [hep-th/9912105] [INSPIRE].

[72] I. Jack and S. Parsons, $O(d, d)$ invariance at two loops and three loops, Phys. Rev. D 62 (2000) 026003 [hep-th/9911064] [INSPIRE].

[73] A.A. Tseytlin, Duality symmetric closed string theory and interacting chiral scalars, Nucl. Phys. B 350 (1991) 395 [inSPIRE].

[74] M. Roček and A.A. Tseytlin, Partial breaking of global D $=4$ supersymmetry, constrained superfields, and three-brane actions, Phys. Rev. D 59 (1999) 106001 [hep-th/9811232] [INSPIRE].

[75] E. Abdalla, M.C.B. Abdalla and M. Gomes, Anomaly in the Nonlocal Quantum Charge of the $\mathbb{C P}^{n-1}$ Model, Phys. Rev. D 23 (1981) 1080.

[76] E. Abdalla, M. Forger and M. Gomes, On the Origin of Anomalies in the Quantum Nonlocal Charge for the Generalized Nonlinear $\sigma$ Models, Nucl. Phys. B 210 (1982) 181 [INSPIRE].

[77] J.M. Evans, D. Kagan and C.A.S. Young, Nonlocal charges and quantum integrability of sigma models on the symmetric spaces $\mathrm{SO}(2 n) / \mathrm{SO}(n) \times \mathrm{SO}(n)$ and $\mathrm{Sp}(2 n) / \mathrm{Sp}(n) \times \mathrm{Sp}(n)$, Phys. Lett. B 597 (2004) 112 [hep-th/0404003] [INSPIRE].

[78] J.M. Evans, D. Kagan, N.J. MacKay and C.A.S. Young, Quantum, higher-spin, local charges in symmetric space sigma models, JHEP 01 (2005) 020 [hep-th/0408244] [INSPIRE].

[79] A.V. Litvinov, Integrable $\mathfrak{g l}(n \mid n)$ Toda field theory and its sigma-model dual, arXiv: 1901.04799 [INSPIRE].

[80] D. Bykov, The worldsheet low-energy limit of the $A d S_{4} \times \mathbb{C P}^{3}$ superstring, Nucl. Phys. B 838 (2010) 47 [arXiv: 1003.2199] [INSPIRE].

[81] B. Basso and A. Rej, On the integrability of two-dimensional models with $\mathrm{U}(1) \times \mathrm{SU}(N)$ symmetry, Nucl. Phys. B 866 (2013) 337 [arXiv:1207.0413] [INSPIRE].

[82] M. Gomes, E. Abdalla and M.C.B. Abdalla, On the Nonlocal Charge of the $C P^{(N-1)}$ Model and Its Supersymmetric Extension to All Orders, Phys. Rev. D 27 (1983) 825 [inSPIRE].

[83] D. Kagan and C.A.S. Young, Conformal sigma-models on supercoset targets, Nucl. Phys. B 745 (2006) 109 [hep-th/0512250] [INSPIRE].

[84] A. Babichenko, Conformal invariance and quantum integrability of sigma models on symmetric superspaces, Phys. Lett. B 648 (2007) 254 [hep-th/0611214] [INSPIRE].

[85] R. Gilmore, Lie Algebras and Some of Their Applications, Dover, (2005).

[86] A. Salam and J.A. Strathdee, On Kaluza-Klein Theory, Annals Phys. 141 (1982) 316 [INSPIRE]. 
[87] L. Castellani, On $G / H$ geometry and its use in $M$ theory compactifications, Annals Phys. 287 (2001) 1 [hep-th/9912277] [INSPIRE].

[88] G. Bonneau, G. Valent and F. Delduc, Renormalization Properties Of Bosonic Nonlinear Sigma Models Built On Compact Homogeneous Kahler Manifolds, Phys. Lett. B 196 (1987) 456 [INSPIRE].

[89] C. Becchi, A. Blasi, G. Bonneau, R. Collina and F. Delduc, Renormalizability and Infrared Finiteness of Nonlinear $\sigma$ Models: A Regularization Independent Analysis for Compact Coset Spaces, Commun. Math. Phys. 120 (1988) 121 [INSPIRE].

[90] A.V. Bratchikov, Renormalization properties of two-dimensional homogeneous symplectic sigma models, Mod. Phys. Lett. A 7 (1992) 2229 [INSPIRE]. 